HD-THEP-00-15

hep-ph/0003122

\title{
Electroweak Baryogenesis: Concrete in a SUSY Model with a Gauge Singlet
}

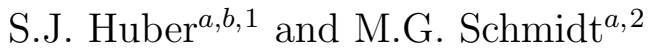 \\ ${ }^{a}$ Institut für Theoretische Physik, Philosophenweg 16, D-69120 Heidelberg, Germany \\ ${ }^{b}$ Bartol Research Institute, University of Delaware, Newark, DE 19716, USA
}

\begin{abstract}
SUSY models with a gauge singlet easily allow for a strong first order electroweak phase transition (EWPT) if the vevs of the singlet and Higgs fields are of comparable size. We discuss the profile of the stationary expanding bubble wall and CP-violation in the effective potential, in particular transitional CP-violation inside the bubble wall during the EWPT. The dispersion relations for charginos contain CP-violating terms in the WKB approximation. These enter as source terms in the Boltzmann equations for the (particle-antiparticle) chemical potentials and fuel the creation of a baryon asymmetry through the weak sphaleron in the hot phase. This is worked out for concrete parameters.
\end{abstract}

\footnotetext{
${ }^{1}$ shuber@udel.edu

${ }^{2}$ m.g.schmidt@thphys.uni-heidelberg.de
} 


\section{Introduction}

Different from models starting at the Grand Unified scale, the ingredients for a creation of the baryon asymmetry of the universe in a first order electroweak phase transition (PT) have a fair chance to be tested experimentally in the near future. Besides nonequilibrium the other two necessary criteria of Sakharov for baryogenesis, CP and baryon number violation are naturally fulfilled by the $\mathrm{CP}$ violating bubble wall of a first order PT. The bubble wall separates the hot symmetric phase with rapid sphaleron transitions from the Higgs phase, where sphaleron transitions are suppressed by $\exp (v(t) / T)$. However it turned out that the electroweak Standard Model (SM) does not have a strong first order PT, indeed there is a crossover behavior and no PT at all for Higgs masses beyond the present experimental bound [1]. Furthermore, CP-violation by the CKM matrix is very small. In spite of tremendous successes of the SM it is commonly believed that it has to be embedded / enlarged to a more fundamental theory. Supersymmetry is supposed to be an important facet of such a theory but it is still not backed by experiments. In concrete SUSY models, in order to have a strong first order PT one has to strengthen the (one loop) " $\varphi$ " term of the effective potential or to have such a term already on the tree level. In the minimal SUSY extension of the SM, the MSSM, the superpartner of the right-handed top - with a mass below that of the top - gives such a strong $\varphi^{3}$ loop correction. With a negative SUSY breaking scalar mass it is almost massless in the hot phase and has a strong Yukawa coupling to the Higgs fields. This is confirmed by detailed analytical [2] and lattice calculations [3], showing a strong first order PT even for Higgs masses as large as $110 \mathrm{GeV}$ just beyond the experimental bound for MSSM Higgses. There are also possible CP-violating phases in the Higgs Lagrangian if 1-loop effects are taken into account [4]. They are restricted much by the measurements of the neutron electric dipole moment unless one invokes special conditions. A recent investigation of a possible spontaneous CP-violation just in the bubble wall at the temperatures of the PT gave negative results [5].

In this paper[ we want to discuss baryogenesis in a strong first order electroweak PT (EWPT) in much detail starting from a model where all the three criteria given above can be fulfilled without much problem. This is a supersymmetric model with an additional gauge singlet superfield. In its original form (NMSSM) is was designed to substitute the problematic $\mu \mathrm{H}_{1} \mathrm{H}_{2}$ term of the MSSM superpotential by a coupling $\mathrm{SH}_{1} \mathrm{H}_{2}$ [6]

$$
W_{Z_{3}}=\lambda S H_{1} H_{2}+\frac{k}{3} S^{3}
$$

with a $Z_{3}$-symmetry whose spontaneous breaking causes dangerous domain walls in the early cosmos [7]. The soft SUSY breaking potential contains a term $\lambda A_{\lambda} S H_{1} H_{2}$ which can act as a $\varphi^{3}$ term if both the vevs of the Higgses and of the singlet are of the same order of magnitude [8]. But in a $Z_{3}$-symmetric model with universal SUSY breaking this turns out not to be possible if one wants to have a reasonable spectrum of particles.

In agreement with ref. [9] we thus add further terms to the superpotential which now obtains the form

$$
W=\lambda S H_{1} H_{2}+\frac{k}{3} S^{3}+\mu H_{1} H_{2}+r S
$$

\footnotetext{
${ }^{3}$ whose content was presented at the TMR network meeting Finite Temperature Phase Transitions in Particle Physics in Korfu, September 1999.
} 
and is not $Z_{3}$-symmetric anymore. The soft SUSY breaking Lagrangian contains scalar masses, gaugino masses and $A$-terms

$$
\mathcal{L}_{A}^{\text {soft }}=\lambda A_{\lambda} S H_{1} H_{2}+\frac{k}{3} A_{k} S^{3}+Y_{e} A_{e} \tilde{e}^{c} \tilde{l} H_{1}+Y_{d} A_{d} \tilde{d}^{c} \tilde{q} H_{1}+Y_{u} A_{u} \tilde{u}^{c} \tilde{q} H_{2}+\text { h.c. }
$$

Thus we have reintroduced a $\mu$-term and we have the associated fine-tuning problem known from the MSSM [10]. Additionally, there is the danger of quadratically divergent singlet tadpoles [11, [2]. Such tadpole diagrams require three ingredients: (i) a singlet field (ii) non-renormalizable interactions and (iii) soft SUSY breaking terms. (i) Giving the "singlet" a charge under some discrete symmetry one can remove the tadpoles from the very beginning. But the discrete symmetry cannot be exact in order to avoid unacceptable domain walls at the EWPT [13. (ii) In refs. 112, 14 models with gauged R-symmetry or duality symmetry, both broken at some superheavy scale have been proposed to forbid dangerous non-renormalizable operators. On the renormalizable level these models typically have no discrete symmetries, and therefore are not plagued by the domain wall problem. While such models do not solve the $\mu$-problem, they are still very interesting with respect to Higgs phenomenology and the EWPT. For that reason we will study this type of singlet model in the following. (iii) Another way to evade the tadpole divergences restricts the soft SUSY breaking terms. "Gauge mediated SUSY breaking" (GMSB) in the context of singlet models does not have domain wall problems. A $\mu$ parameter is generated by radiative corrections and the singlet vev [15]. However one of the special properties of GMSB models seems to be the strong suppression of $A$-terms. These however also contain the $\varphi$-type term responsible for a strong first order PT.

In order not to have too many parameters we use universality of SUSY breaking at the GUT scale and run renormalization group equations to get down to the electroweak scale. With such a Lagrangian and adding the temperature corrections we can demonstrate [16] that one can easily get a strong first order PT for Higgs masses as high as $115 \mathrm{GeV}$ and superpartner masses beyond present experimental limits. The effective potential in $H_{1}$, $\mathrm{H}_{2}$ and $\mathrm{S}$ can be used to derive differential equations for the bubble wall profile and for varying $\mathrm{CP}$-violating phases in the bubble wall arising from constant explicit phases in the theory or, more interesting, from spontaneous CP-violation. This is described in sections 4 and 5. As worked out in section 6, in the WKB approximation the CP-violating phases in the bubble wall create terms in the dispersion relations which differ between particles and anti-particles. This gives rise to a driving term in the Boltzmann equation for the difference of particle and anti-particle chemical potentials, in particular for left-handed quarks and their superpartners. This difference is converted into a baryon asymmetry by the hot phase sphalerons in front of the proceeding bubbles.

\section{The model}

Weak scale supersymmetric models have many unknown parameters related to supersymmetry breaking. A considerable part of this parameter space is excluded because of FCNC or the appearance of charge and color breaking vacua. These problems are partially evaded by imposing universality of the soft terms at the GUT scale, $M_{G U T} \sim 2.6 \cdot 10^{16}$ $\mathrm{GeV}$, where the SM gauge couplings unify. At the GUT scale there is a common gaugino mass $M_{0}$, a universal scalar mass squared $m_{0}^{2}$ and a universal trilinear coupling $A_{0}$. Thus, 
universal SUSY breaking drastically reduces the parameters of the model to

$$
y_{t 0}, \lambda_{0}, k_{0}, M_{0}, A_{0}, m_{0}^{2}, \mu_{0}, r_{0}, B_{0} .
$$

The subscript "0" indicates that the masses and couplings are evaluated at the GUT scale. $y_{t}$ denotes the top Yukawa couplingf, and $\mu B H_{1} H_{2}$ is the soft Higgs mass term corresponding to the $\mu$-term in the superpotential. Since the masses of the top quark and the $Z$-boson mass are known, "only" seven parameters of (2.3) are independent.

To evolve the parameters from the GUT scale to the weak scale we use the renormalization group equations (RGEs) in the 1-loop approximation. For the $Z_{3}$-symmetric case the relevant RGEs have already been given in ref. [17]. Defining $t=\ln Q^{2}$ the $Z_{3}$-breaking terms $\mu, r$ and $B$ are easily included with help of ref. [18]

$$
\begin{aligned}
\frac{d}{d t} \mu & =\frac{1}{32 \pi^{2}} \mu\left(3 y_{t}^{2}+2 \lambda^{2}+2 k^{2}-3 g_{2}^{2}-g_{1}^{2}\right) \\
\frac{d}{d t} r & =\frac{1}{16 \pi^{2}} r\left(\lambda^{2}+k^{2}\right) \\
\frac{d}{d t} B & =\frac{1}{16 \pi^{2}}\left(2 \lambda^{2} B+3 y_{t}^{2} A_{t}+2 \lambda^{2} A_{\lambda}+3 g_{2}^{2} M_{2}+g_{1}^{2} M_{1}\right) .
\end{aligned}
$$

Even with the simple universal pattern of soft breaking terms we are left with a 9dimensional parameter space. Additionally, we must satisfy two constraints coming from the Z-boson and top quark masses. In the literature (see e.g. ref. [19]), usually "random shooting" is applied to deal with such a situation. This means randomly chosen sets of GUT scale parameters are evolved to the electroweak scale, where their phenomenological implications are investigated. Although the physical Z-boson mass can easily be reproduced by an appropriate rescaling of the dimensionful parameters, one typically is still plagued by some light unobserved SUSY particles or an unphysical top quark mass. Therefore this procedure is rather inefficient. Furthermore, random shooting only provides statistical averages and correlations. To avoid these shortcomings we use the more systematic approach which we introduced in ref. [16]. It allows the elimination of $\mu, r$ and $B$ by the Higgs and singlet vevs, while maintaining universal SUSY breaking. It relies on the observation that the $Z_{3}$-breaking terms $\mu, r$ and $B$ do not enter the RGEs for the remaining parameters. They can therefore be calculated without specifying the former ones. Our procedure consists of the following steps and is also sketched in fig. 1:

- Fix the values of $x \equiv\langle S\rangle, \tan \beta=v_{2} / v_{1}, \lambda$ and $k$ at the weak scale. The choice of $\tan \beta$ automatically fixes $y_{t}$ by the relation $y_{t} v \sin \beta=m_{\text {top }}(=175 \mathrm{GeV})$.

- Evolve $y_{t}, \lambda$ and $k$ to the GUT scale.

- Choose a set of the parameters $M_{0}, A_{0}$ and $m_{0}^{2}$ at the GUT scale.

- Run all parameters down to the weak scale, with exception of $\mu, r$ and $B$ whose initial conditions have not yet been specified at this point.

- At the weak scale calculate $\mu, r$ and $B$ by using the saddle point conditions for the 1-loop Higgs potential $\partial_{I} V\left(H_{1}, H_{2}, S\right)=0\left(I=H_{1}, H_{2}, S\right)$ at $H_{1}^{-}=H_{2}^{+}=0$, $H_{1,2}^{0}=v_{1,2}, S=x$.

\footnotetext{
${ }^{4}$ We neglect the Yukawa couplings of the leptons and light quarks. This approximation is well justified in the range of small and mediate $\tan \beta$ which we consider in the following.
} 


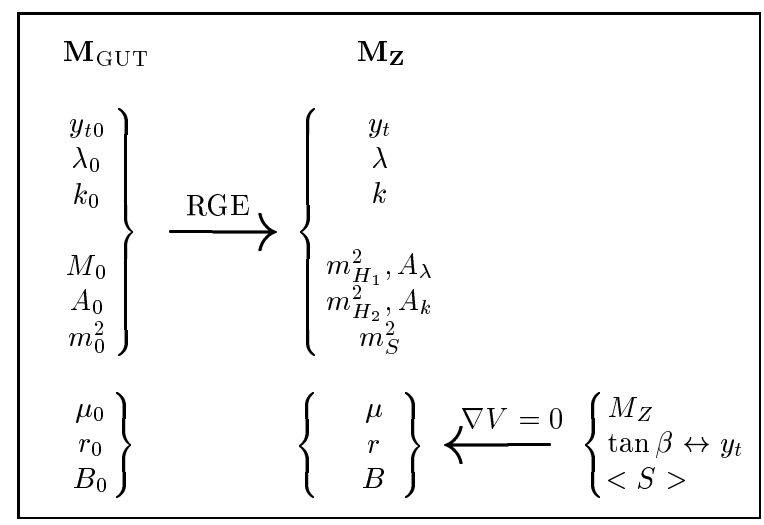

Figure 1: Sketch of our procedure to fix the weak scale parameters using RGEs and saddle point conditions.

Finally one can use the corresponding RGEs to determine the values of $\mu, r$ and $B$ at the GUT scale. Appropriate values of $\mu_{0}, r_{0}$ and $B_{0}$ can always be found because of the effectively linear structure of eqs. (2.4). Our procedure has the additional benefit that the constraints coming from the top quark and Z-boson masses, $M_{Z}^{2}=\left(g_{1}^{2}+g_{2}^{2}\right)\left(v_{1}^{2}+v_{2}^{2}\right) / 2$, are automatically built in. This reduces the number of free parameters by two so we are finally left with the 7-dimensional parameter space

$$
\tan \beta, x, \lambda, k, M_{0}, A_{0}, m_{0}^{2} \text {. }
$$

Of course, not every parameter set leads to a viable phenomenology. The constraints on the model parameters are related to the particle spectrum and the vacuum structure. In the elimination procedure discussed before we assumed that the extremum parametrized by $v, \tan \beta$ and $x$ is indeed the global minimum of the scalar potential. Thus we must (numerically) verify that there are no deeper minima in $V\left(H_{1}, H_{2}, S\right)$. If there appears a deeper minimum the parameter set has to be discarded.

Additional constraints arise from the required absence of charge and color breaking minima (CCB minima) deeper than the standard minimum, i.e. from the absence of squark and slepton vevs. We check slepton and squark vevs induced by large trilinear couplings 20,21]. Furthermore, there are CCB minima which come from negative scalar mass squares, typically $m_{H_{2}}^{2}$. These dangerous directions involve Higgs, squark and slepton fields ("UFB" directions). The condition for such a minimum not to be deeper than the standard minimum implies a lower limit on the ratio $m_{0}^{2} / M_{0}^{2}$ of $\mathcal{O}(1)$ 222. However, the decay rate of the standard vacuum into a minimum in the UFB direction is usually negligible compared to the age of the universe. We therefore allow for a meta-stable standard vacuum with respect to the UFB directions and disregard the corresponding constraints.

Up to now, no superpartners of the SM particles have been detected. From the experimental lower limits on the SUSY particle masses [23] various constraints on the parameter space can be derived. The experimental limit on the chargino mass $M_{\tilde{\chi}_{1}^{ \pm}}>90 \mathrm{GeV}$ [24] translates into bounds on the universal gaugino mass and the $\mu$ parameter

$$
\left|M_{0}\right| \gtrsim 100 \mathrm{GeV}, \quad|\mu+\lambda x| \gtrsim 80 \mathrm{GeV}
$$


(a)

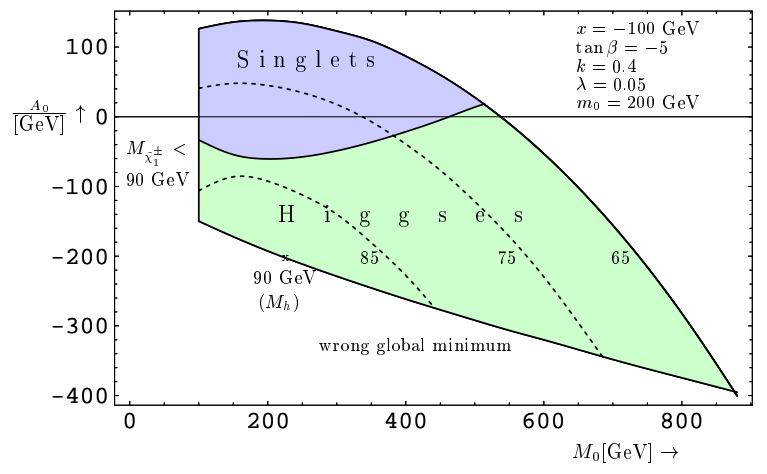

(b)

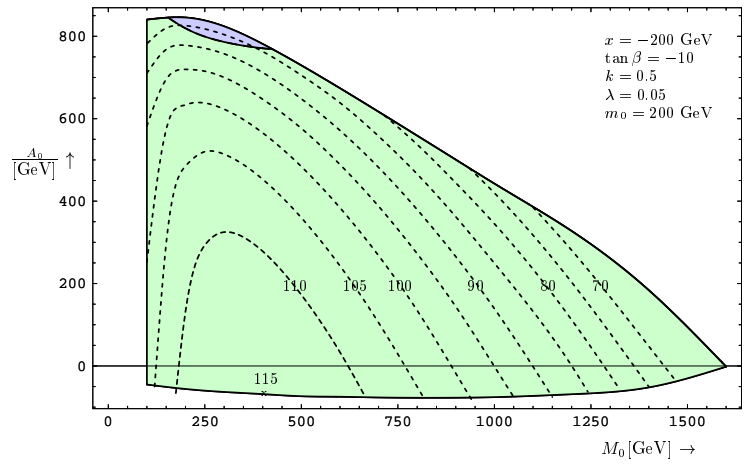

Figure 2: Scan of the $M_{0}-A_{0}$ plane for two different sets of $(x, \tan \beta, k)$. The colored regions represent the phenomenologically viable range of the parameters before cuts from the Higgs boson search are applied. The dashed lines are curves of constant mass of the lightest CP-even Higgs boson. In the blue (green) areas, the lightest Higgs boson is predominantly a singlet (Higgs) state.

If the bound on $M_{0}$ is satisfied the gluino mass $M_{3}$ is automatically above its experimental limit [23]. In the case of $m_{0}>100 \mathrm{GeV}$ also the squark and slepton masses are compatible with the experimental data.

Of particular importance are the properties of the lightest neutral CP-even 'Higgs' mass eigenstate $h$, which is a mixture of the Higgses and the singlet. If $h$ has a large singlet content its coupling to the Z-boson and thus its production cross section at LEP is significantly reduced [25]. Parameter sets (2.5) cannot be ruled out by simply calculating the lightest Higgs mass $M_{h}$. For example, if the Higgs production cross section is reduced by a factor of 10 compared to the SM, Higgs masses down to about $60 \mathrm{GeV}$ are still in agreement with the data [26].

In fig. 2 we present a scan of the $M_{0}-A_{0}$ plane for two different sets of $(x, \tan \beta, k)$ [16]. The parameters $M_{0}$ and $A_{0}$ are of particular interest since they determine the values of the trilinear couplings at the weak scale which will play a prominent role in the discussion of the EWPT in section 3. We have taken $m_{0}>100 \mathrm{GeV}$ in order avoid light squarks and sleptons. Choosing a small value of $\lambda$ and a large value of $k$ prevents the appearance of very light Higgs bosons. In fig. 2 $\mathrm{b}$ we increased $\tan \beta$ and the singlet vev to obtain large Higgs masses up to $115 \mathrm{GeV}$. The increase of $M_{h}$ with decreasing $A_{0}$ can be traced to the singlet diagonal entry in the Higgs boson mass matrix which is diminished by the $A_{k}$ contribution. On the same lines the increasing singlet content of the lightest CP-even Higgs state with increasing $A_{0}$ can be understood. In fig. $2 \mathrm{~b}$ this effect is reduced by the larger values of $|x|$ and $k$, which render the singlet state rather heavy.

The most important constraints on $M_{0}$ and $A_{0}$ arise from the chargino mass and from the vacuum structure. The lower bound on $M_{0}$ is a consequence of eq. (2.6). The requirement of the standard minimum being the global minimum of the Higgs potential implies the upper and lower bounds on $A_{0}$ displayed in fig. 2. Constraints from the required absence of $A$-term induced CCB minima are then automatically satisfied. Note that $M_{0}$ is also bounded from above. In fig. 2 we excluded parameter sets which predict $M_{h}<65 \mathrm{GeV}$. This leads to the upper bound on $A_{0}$ in fig. 2a. In fig. 2b no additional constraint arises, because of the larger Higgs masses. On the other hand, if we would 
allow for smaller Higgs masses in fig. 2a, still an upper bound on $A_{0}$ would be implied by the vacuum structure, as is the case in fig. 2pb. Taking into account the data from the Higgs boson search further restricts the upper bounds on $M_{0}$ and $A_{0}$. Roughly, the regions $M_{0}>600 \mathrm{GeV}$ in the $\tan \beta=-5$ set, and $M_{0}>1200 \mathrm{GeV}$ in the $\tan \beta=-10$ set are additionally excluded by the Higgs boson data [26]. These parameter regions are characterized by a very heavy spectrum of SUSY particles. For that reason they are not very promising candidates for the implementation of electroweak baryogenesis anyway, as will be discussed in section 6 .

We close this section by emphasizing some important differences between our model and the $Z_{3}$-symmetric NMSSM, which is usually considered in the literature, e.g. in refs. [19, 25]. By setting $\mu=0$ eq. (2.6) can be translated into a lower bound on the singlet vev, $|x| \gtrsim 125 \mathrm{GeV}$. However, the analysis of the $Z_{3}$-symmetric case carried out in ref. [19] shows that the actual lower bound on $|x|$ is much larger, once all phenomenological constraints are taken into account. Typically one has a singlet vev in the multi-TeV range and couplings $\lambda \ll 1$ and $k \ll 1$, with $\lambda x$ and $k x=\mathcal{O}\left(M_{Z}\right)$. In the next section we will discuss that in such a scenario the singlet is just a spectator during the EWPT, which proceeds more or less in the standard (i.e. MSSM) way. This property additionally motivates our inclusion of the $\mu$-term in the model, while it seems questionable whether the idea of electroweak baryogenesis can be realized in the $Z_{3}$-symmetric NMSSM. An additional peculiarity of the $Z_{3}$-symmetric model is that large $A$-terms are required for successful electroweak symmetry breaking, leading to $A_{0}^{2}>9 m_{0}^{2}$. However, this parameter range is severely constrained from the absence of CCB minima [21]. Some of the special features reported in ref. [19] may be due to the assumption of universal soft SUSY breaking used in that work. It seems questionable, however, if the problematic bound, $|x| \gg M_{Z}$, can be evaded in the case of non-universal soft terms [27], since the required large values of $\lambda$ typically lead to small values of the Higgs mass due to large Higgs singlet mixing. The NMSSM with and without $Z_{3}$ symmetry also have different properties with respect to CP-violation, as will be discussed in section 5 .

\section{Strength of the electroweak phase transition}

Order and strength of the EWPT are central questions in electroweak baryogenesis. Only in the case of a first order phase transition (PT) the associated departure from equilibrium is sufficient to induce a relevant baryon number production. To avoid baryon number washout after the PT the even stronger criterion $v_{c} / T_{c} \gtrsim 1$ has to be satisfied, where $v_{c}$ denotes the Higgs vev at the critical temperature $T_{c}$. A first order phase transition is triggered by cubic terms in the finite temperature effective potential [28]. In the (MS)SM these terms arise from 1-loop thermal corrections of bosons and therefore are small from the very beginning. Thus it is difficult to satisfy $v_{c} / T_{c}>1$. In the NMSSM, on the other hand, trilinear terms enter already the tree-level Higgs potential due to Higgs singlet couplings, leading to a significantly stronger EWPT [8, 9, 16]. These contributions stem from the soft SUSY breaking couplings $A_{\lambda}$ and $A_{k}$, and from the $\mu$-term

$$
\left(\lambda \mu^{*} S+\text { h.c. }\right)\left(\left|H_{1}^{0}\right|^{2}+\left|H_{2}^{0}\right|^{2}\right)+\left(\lambda A_{\lambda} S H_{1}^{0} H_{2}^{0}+\frac{k}{3} A_{k} S^{3}+\text { h.c. }\right) \text {. }
$$

All these trilinear terms explicitly contain the singlet field. Therefore, in order to induce deviations from the (MS)SM behavior also the singlet vev must change during the phase 
transition. Since at the EWPT thermal contributions to the effective potential are of the order $\mathcal{O}\left(T^{4}\right) \sim M_{Z}^{4}$, this requires the mass and the vev of the singlet also to be of the order of the electroweak scale.

At finite temperature the effective potential of the neutral Higgs and singlet fields is modified by the interaction with the hot plasma

$$
V_{T}\left(H_{1}^{0}, H_{2}^{0}, S\right)=V_{\text {tree }}\left(H_{1}^{0}, H_{2}^{0}, S\right)+V^{(1)}\left(H_{1}^{0}, H_{2}^{0}, S\right)+V_{T}^{(1)}\left(H_{1}^{0}, H_{2}^{0}, S\right) .
$$

In the 1-loop zero temperature corrections $V^{(1)}$ we include tops, stops and gauge bosons, while in the 1-loop finite temperature part $V_{T}^{(1)}$ also Higgs bosons, neutralinos and charginos are taken into account. We do not make a high temperature expansion, as some of the particles can be heavy in part of the field space. Rather, $V_{T}^{(1)}$ is evaluated using a spline interpolation between the high and low temperature regions.

We stress that in the range of parameters we will study a strong first order EWPT is the consequence of the tree-level terms in the Higgs potential (3.7). Since the stop mass will turn out to be always larger than $200 \mathrm{GeV}$, the thermally induced cubic terms are too small to account for $v_{c} / T_{c}>1$ [2]. The most important finite temperature effect is the appearance of thermal effective masses

$$
m^{2} \rightarrow m^{2}(T)=m^{2}(T=0)+\text { const } \cdot T^{2},
$$

where the constant encodes the couplings of the Higgs and singlet fields to the particles in the plasma. It is this positive thermal contribution to the Higgs mass that makes the symmetric phase stable at high temperatures, and thus causes the restoration of the electroweak symmetry. Using the complete 1-loop expression rather than the simple high temperature approximation, is just a convenient prescription to handle the decoupling of heavy particles. Because of the dominance of the tree potential, we neglect contributions to the thermal potential stemming from daisy resummation [29, 30] and 2-loop diagrams [2].

In order to determine the strength of the PT one has to compute the critical temperature $T_{c}$. We define $T_{c}$ as being the temperature where the symmetric and the broken minimum of the finite temperature Higgs potential (3.8) become degenerate. We denote the Higgs and singlet vevs in the broken phase by $\left(v_{1 c}, v_{2 c}, x_{c}\right)$. In general, the singlet vev is different from zero even in the symmetric phase. We refer to a PT as being "strongly first order", if it avoids baryon number washout, according to the condition $v_{c} / T_{c}>1$. Since both Higgs fields contribute to the sphaleron energy, $v_{c}$ is given by $v_{c}=\sqrt{2}\left(\left|v_{1 c}\right|^{2}+\left|v_{1 c}\right|^{2}\right)^{1 / 2}$, where the factor of $\sqrt{2}$ is due to our choice of field normalization. Although CP-violation is essential for baryogenesis, we assume CP-conservation in this section, i.e. all vevs, mass parameters and coupling constants are taken real valued. Of course, we have to verify that $V_{T}$ has no deeper CP-violating minimum. Turning on phases much smaller than one would induce only marginal changes in our results.

In this paragraph we study again the two parameter sets already discussed in the context of fig. 2 in section 2. We determine the critical temperature by numerical minimization of $V_{T}$. After having checked that no CP-violating minima are present we disregard the imaginary parts of the Higgs and singlet fields.f We then only have to minimize $V_{T}$ in the real valued fields $\operatorname{Re}\left(H_{1}^{0}\right), \operatorname{Re}\left(H_{2}^{0}\right)$ and $\operatorname{Re}(S)$.

\footnotetext{
${ }^{5}$ Bubble walls in the presence of CP-violation will be discussed in section 5 .
} 
(a)

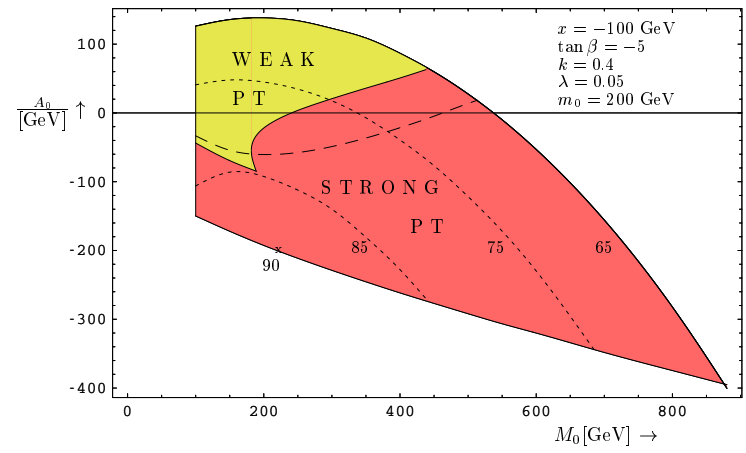

(b)

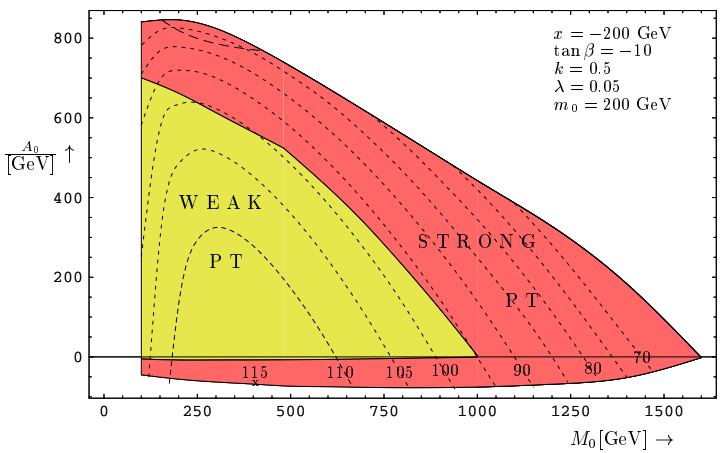

Figure 3: Scan of the $M_{0}-A_{0}$ plane for the two different sets of $(x, \tan \beta, k)$ which have already been considered in fig. 2. In the red (yellow) areas the PT is strongly (weakly) first order, i.e. $v_{c} / T_{c}>1\left(v_{c} / T_{c}<1\right)$. The dotted lines are curves of constant mass of the lightest CP-even Higgs boson. In the regions below (above) the dashed lines the lightest Higgs boson is predominantly a Higgs (singlet) state.

Our investigations of the strength of the PT are summarized in fig. 3 where the regions of strong and weak PT in the $M_{0}-A_{0}$ plane are displayed for the two parameter sets of fig. 2 [16]. In case of $\tan \beta=-5$ and $x=-100 \mathrm{GeV}$ (fig. 3a) the PT is strongly first order in most part of the phenomenologically allowed range of parameters. However, the corresponding Higgs masses up to $90 \mathrm{GeV}$ are compatible with the experimental Higgs mass bounds only because of the reduced Higgs production cross section due to Higgs singlet mixing. In the parameter set of fig. $3 \mathrm{~b}$ we increased $|\tan \beta|$ and $|x|$ in order to obtain larger Higgs masses. As a consequence, the region of weakly first order PT is enlarged. However, a strong PT occurs still for a wide range of the parameters, while Higgs masses up to $115 \mathrm{GeV}$ are consistent with $v_{c} / T_{c}>1$.

In contrast to the SM or the MSSM where the PT definitely becomes weaker with increasing Higgs masses the situation in the NMSSM is more complicated. Larger Higgs masses can be related to a stronger phase transition. This may happen for example if the broken and symmetric minima of the effective potential are almost degenerate already at zero temperature. The strong PT at negative $A_{0}$ and $M_{h}>100 \mathrm{GeV}$ in fig. 3b results precisely from this effect.

Our results [16] are in reasonable agreement with those of ref. [9], where the strength of the EWPT in the general NMSSM has also been studied. The authors found that about $50 \%$ of their parameter sets were compatible with $v_{c} / T_{c}>1$, and also advocated for $x \sim v$ in order to obtain a strong phase transition. While in ref. [9] no restrictions with respect to the SUSY breaking were made, we demonstrated that even with universal SUSY breaking a strongly first order PT is quite natural in the general NMSSM. Furthermore, we used updated (more restrictive) experimental bounds to constrain the parameter space. A further difference comes from the procedure used in scanning the parameter space. In ref. [9] random shooting was applied, which only allowed for statistical statements. Moreover, from the initially tested 105000 parameter sets just $2 \%$ gave a viable zero temperature phenomenology, leaving merely about 2000 sets for the study of the PT. This again demonstrates the usefulness of the systematic procedure for scanning the parameter space, which we described in section 2. 


\section{Shape of the phase boundary}

In the previous section we defined $T_{c}$ as being the temperature where the symmetric and the broken minimum of $V_{T}$ become degenerate. However, tunneling with formation of bubbles of the broken phase starts at some lower "nucleation" temperature, when the symmetric phase is already meta-stable. The bubble wall profile will crucially enter the calculation of the baryon production during the PT which will be discussed in section

6. Here we concentrate on CP-conserving bubble walls. The very important case of $\mathrm{CP}$-violating wall profiles will be discussed in the next section.

At high temperatures the probability for thermal tunneling is proportional to $e^{-S_{3} / T}$, where $S_{3}$ is the three-dimensional action of the static field configuration $\Phi(\vec{x})$ describing tunneling [31]. Here $\Phi$ collectively denotes the Higgs and singlet fields. Assuming spherical symmetry the bubble configuration ("critical bubble") obeys the equation of motion

$$
\frac{d^{2} \Phi}{d r^{2}}+\frac{2}{r} \frac{d \Phi}{d r}-\frac{\partial}{\partial \Phi} V_{T}(\Phi)=0
$$

Further simplifications of eq. (4.10) are justified if the tunneling occurs between two almost degenerate minima of the potential with an energy difference $\Delta V_{T}$ much smaller than the height of the potential barrier. In such a case the radius of the bubble becomes much larger than the thickness of the bubble wall, which thus is referred to as "thin wall limit". Neglecting therefore the $d \Phi / d r$ term in (4.10) we are left with

$$
\frac{d^{2} \Phi}{d z^{2}}-\frac{\partial}{\partial \Phi} V_{T}(\Phi)=0
$$

We have replaced the spatial coordinate $r$ by $z$, indicating that the solution to (4.11) may be viewed as a planar domain wall with translational invariance in the directions perpendicular to the $z$ axis. During the period of stationary expansion the pressure induced by the energy difference of the minima, $\Delta V_{T}$, is compensated by friction [67, 68]. In the following we model this effect by taking the effective potential at the critical temperature.

Notice that (4.11) is just the classical equation of motion of a particle moving from one maximum of the turned around potential $-V(\Phi)$ to the other, where is comes at rest. In this picture $z$ takes the role of the time coordinate and $\Phi$ represents the configuration space variable. Obviously, this a very delicate process, especially if more than one scalar field is involved. Small changes of the initial conditions lead to a completely different shape of the trajectory.

For a general effective potential the bubble wall equations have to be solved numerically. In the case of only one scalar field the so called "overshooting undershooting procedure" can by applied: The initial value $\Phi\left(r_{0}\right)$ is tuned such that the trajectory approaches $\Phi=\Phi_{\text {sym }}$ in the limit $r \rightarrow \infty$, which then gives the bubble shape. This procedure can be used for the critical bubble as well as for the domain wall configuration.

The situation is completely different once there are additional directions in field space. Although in principle the shooting procedure is still applicable, in practice the required high accuracy of the initial conditions cannot be achieved. Thus one has to devise other numerical methods [5, 33 37]. They determine the bubble wall configuration as the minimum of a functional $\mathcal{F}[\Phi]$ which is built from the squared equations of motion 35. Details concerning the numerical algorithms used to minimize $\mathcal{F}$ on a lattice are given in 
refs. 33, 35]. It turns out that the minimization procedure is reliable only if the starting configuration, i.e. the ansatz for the wall shape, differs not too much from the actual solution. Otherwise one keeps being stuck to local minima, which arise from discretizing the functionals on a lattice [33]. Thus minimization starting from an arbitrary initial configuration is not possible up to now, and finding an appropriate ansatz is very important. In the case of the domain wall the kink solution fits the exact bubble shape reasonably well, especially if every field is allowed for having its own wall thickness

$$
\phi_{i}(z)=\frac{v_{i}}{2}\left(1-\tanh \left(\frac{z-\delta_{i}}{L_{i}}\right)\right) .
$$

In general, different off-sets $\delta_{i}$ which shift the fields against each other are possible as well. For the domain wall case the mechanical analogue to energy conservation, $E(z)=$ $\frac{1}{2}(d \Phi / d z)^{2}-V(\Phi)=$ const, provides a very sensitive criterion to check the quality of the numerically obtained solution.

In general, the numerical methods developed in refs. [5, 33 36] are inevitable to determine the bubble shape in multi-field models. But if the problem is effectively one dimensional some "improved shooting method" can still be applied. The investigation of CP-conserving bubble wall shapes in the MSSM [33, 35] revealed that for realistic Higgs masses the variation of $\tan \beta$ in the bubble is very small, $\delta \beta \sim \mathcal{O}\left(10^{-2}-10^{-3}\right)$. Thus the bubble is very accurately described by taking only the combination of the Higgs fields, $H$, which corresponds to the direction of the broken minimum. Simple shooting along this direction provides excellent values for the wall thickness, the surface tension or the action of the critical bubble. Even the small variation in $\beta$ can be reliably determined by minimizing the effective potential along the direction perpendicular to $H$. The line which connects these minima gives a good approximation to the trajectory in field space the actual bubble solution is corresponding to. This can be checked by using the "energy conservation" criterion. The kink ansatz turns out to be a good fit for bubble profile.

In case of the NMSSM the straight connection between the broken and the symmetric minimum is no longer a reasonable approximation to the true bubble wall trajectory. Ignoring the variation of $\tan \beta$, which turns out to be small and hence can be included afterwards, we effectively have a two field system, $H$ and $S$. In a large part of the parameter space (as long as $A_{0}$ is significantly above its lower bound shown in fig. 2) the effective potential is characterized by a distinct smooth ridge which connects the symmetric and the broken minimum. This ridge $S=F(H)$ can be determined by minimizing the potential along the direction perpendicular to the straight connection of the symmetric and broken minima. [ Using $V_{T}(H, F(H))$ the set of bubble wall equations (4.11) reduces to only one differential equation which can again be solved via the shooting method. However, not every function $F$ is a possible candidate for the actual bubble wall. Since $d H / d z$ vanishes in the symmetric and broken phase, integrating the bubble wall equation results in the constraint

$$
\int_{0}^{H_{\mathrm{brk}}} d H \frac{\partial}{\partial H} V_{T}(H, F(H))=0 .
$$

\footnotetext{
${ }^{6}$ Numerically, the following method leads to better results: Determine the saddle point along the ridge, i.e. $\partial_{H} V_{T}=\partial_{S} V_{T}=0$. The ridge is then defined as the trajectory of an over-damped particle rolling from the saddle point down to the (symmetric or broken) minimum, satisfying $d \Phi / d z+\partial_{\Phi} V_{T}=0$.
} 

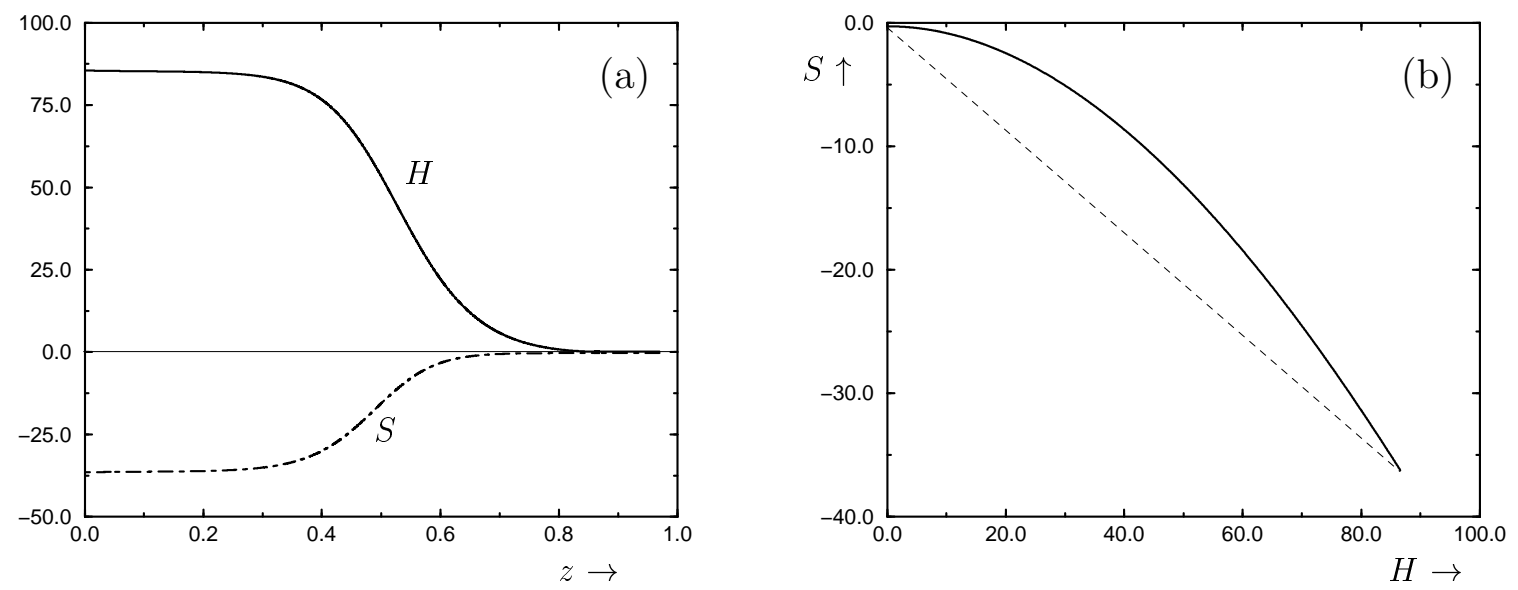

Figure 4: (a): Bubble wall profile at the critical temperature for the parameter set of fig. 2 a with $M_{0}=300 \mathrm{GeV}$ and $A_{0}=0$. (b): The corresponding trajectory in the $H$ $S$ plane (solid line) and the straight connection between the symmetric and the broken minimum (dashed line). (All units in GeV.)

In order to evade this inconsistency we deform our ansatz by $\tilde{F}(H)=F(H)+f H\left(H-v_{c}\right)$, where the free parameter $f$ is fixed by eq. (4.13). This ansatz is motivated by the mechanical analogue of the bubble wall problem: if a ball rolls from one maximum of $-V_{T}$ to the other, it moves a bit below the ridge in order to compensate for the centrifugal force. On half way between the two maxima the centrifugal force is most efficient and the trajectory $\tilde{F}$ deviates maximally from the ridge $F$. In practice, $\tilde{F}-F$ is small compared to the deviation of $F$ from the straight line.

Instead of expressing $S$ in terms of $\tilde{F}(H)$ one could just as well eliminate $H$ by $\tilde{F}^{-1}(S)$. If $\tilde{F}$ corresponds to the trajectory of the actual bubble configuration the two prescriptions are equivalent. For a general $\tilde{F}$ the two prescriptions will lead to different results for the wall profile. The difference between the two solution indicates the quality of the chosen $\tilde{F}$. We emphasize that although several steps are necessary to compute the bubble wall shape via this improved shooting approach, the required numerics is fairly simple. It can by carried out using computer algebra systems like Maple or Mathematica. The results are competitive to those obtained with the sophisticated minimization algorithms of refs. 33, 35], which on the other hand were very helpful to check the validity of our approximations.

Let us discuss a specific example from the parameter set of fig. 2a with $M_{0}=300$ $\mathrm{GeV}$ and $A_{0}=0$. It corresponds to a lightest Higgs mass $M_{h}=80 \mathrm{GeV}$, which because of the reduced coupling to the Z-boson is compatible with the experimental data. We have $T_{c}=109 \mathrm{GeV}$ and $v_{c} / T_{c}=1.12$, i.e. the washout of baryon number after the phase transition is avoided. Using our improved shooting method we find the bubble wall profile displayed in fig. 价. In fig. \#b we show the corresponding trajectory in field space which considerable deviates from a straight line. Fitting the numerical solutions by the kink ansatz (4.12) we obtain the wall thicknesses for the Higgs and singlet fields, $L_{h}=0.13$ $\mathrm{GeV}^{-1}=14 / T_{c}$ and $L_{s}=0.10 \mathrm{GeV}^{-1}=11 / T_{c}$, respectively. For the surface tension we find $\sigma=46300 \mathrm{GeV}^{3}$. The energy conservation check gives $\Delta E=6000 \mathrm{GeV}^{4}$ which has to be compared to the barrier height $V_{b} \sim 125000 \mathrm{GeV}^{4}$. This is the same level of 

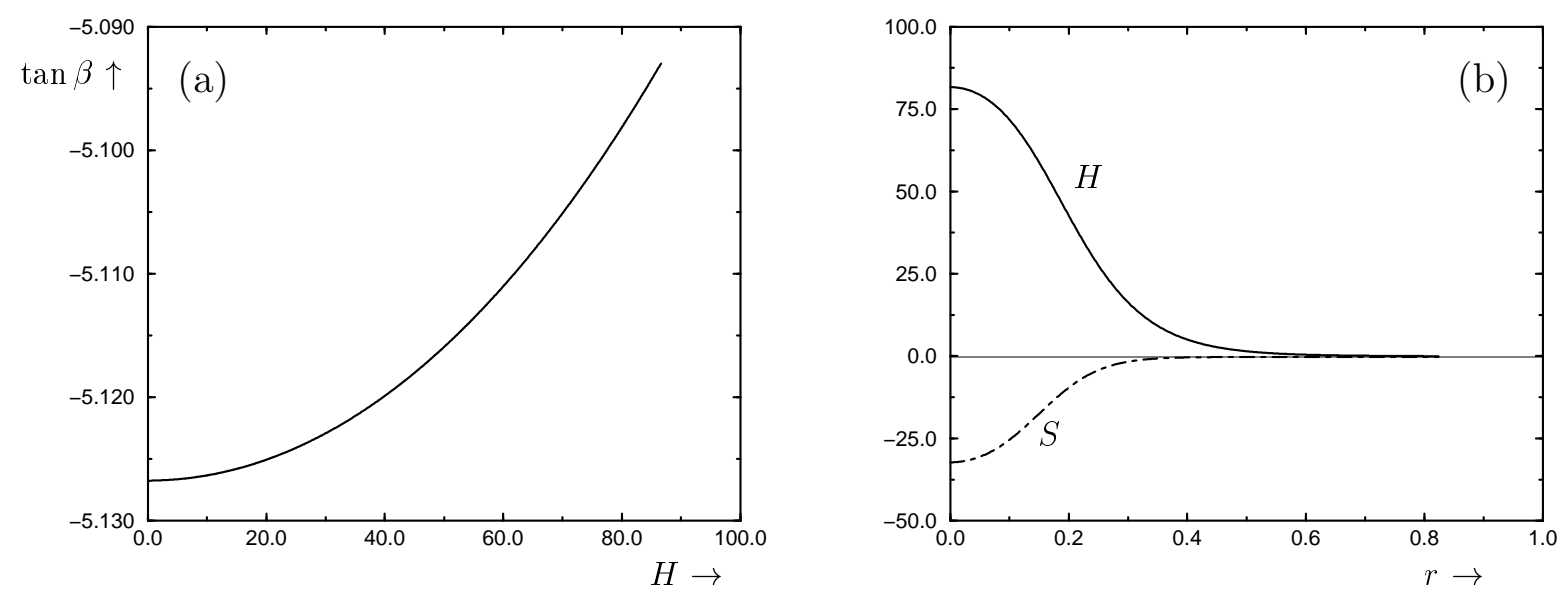

Figure 5: (a): Variation of $\tan \beta$ in the bubble wall at the critical temperature for the parameter set of fig. 1 . (b): Shape of the critical bubble for the same parameter set at $T=108.5 \mathrm{GeV}$. (Units in $\mathrm{GeV}$.)

accuracy [37] which is accessible in the minimization approach of ref. [33]. On the other hand, taking the simplest ansatz for $\tilde{F}$, the dashed straight line in fig. Ab, results in $L_{h}=L_{s}=8 / T_{c}$ and $\sigma=82200 \mathrm{GeV}^{3}$, which is already off by about a factor of two.

Up to now we neglected the variation of $\tan \beta$ in the bubble wall which has a strong impact on some sources for baryogenesis, as will be discussed in section 6 . In order to include this effect in our calculation we simply minimize the effective potential in the direction orthogonal to $H$ while keeping $H$ and $S$ fixed, implicitly assuming that it is only a small perturbation to the trajectory $\tilde{F}(H)$. In fig. $5 \mathrm{a}$ we show the variation of $\tan \beta$ for the bubble wall of fig. 4 . We obtain $\delta \beta=1.2 \times 10^{-3}$, i.e. the assumption of $\delta \beta$ being a small perturbation is very well justified. Our result is in complete agreement [37] with the one computed by using the minimization technique of ref. [33]. Moreover, we find that the variation of $\tan \beta$ in the NMSSM and MSSM [35] are of the same order of magnitude. Particularly, the singlet field provides no additional sources for $\delta \beta$ which can be traced to its equal couplings to both Higgs fields.

Finally, our improved shooting method can be used to determine the shape of the critical bubble (4.10). The first bubbles nucleate when the action of the critical bubble satisfies $S_{3}\left(T_{n}\right) / T_{n} \sim 130-140$ [32]. In the computation of the $T_{n}$ the small tan $\beta$ variation in the wall can be safely ignored. Considering again the parameter set of fig. 4 we obtain the bubble configuration shown in fig. $5 \mathrm{~b}$, where $T=108.5 \mathrm{GeV}$. Since $T_{c}=109.2 \mathrm{GeV}$ the super-cooling amounts to $0.7 \mathrm{GeV}$, the same order of magnitude as in the MSSM [35. Notice that the wall shape is still very similar to a kink solution (4.12), which is cut off at $r=0$. Calculating the corresponding action we get $S_{3} / T=134$. Thus we are at the nucleation temperature. Note that the thin wall approximation $S_{3}=\frac{16 P i}{3} \sigma^{3} /\left(\Delta V_{T}\right)^{2}$, leading to $S_{3} / T=94$ is not very accurate in this case. Here $\Delta V_{T}=4.04 \times 10^{5} \mathrm{GeV}^{4}$ is the potential barrier. Since in our example bubble radius and wall thickness are of comparable size this difference is not surprising.

We emphasize that our method to determine bubble shapes is restricted to cases where the bubble trajectory is associated with a smooth ridge in the effective potential, which correspond to values of $A_{0}$ significantly above its lower bound. Otherwise one is referred 
to the sophisticated minimization methods of refs. [33, 35, 36].

\section{CP-violating bubble walls}

Having established a strongly first order EWPT in the NMSSM, successful electroweak baryogenesis still depends crucially on the available amount of CP-violation. In models containing two Higgs doublets, such as the MSSM or the NMSSM, complex vevs of the Higgs (and singlet) fields provide additional sources of CP-violation. Complex vevs can either arise spontaneously or are induced by explicitly CP-violating couplings. In this section we discuss their impact on bubble wall profiles in the NMSSM. Most interestingly, we find that $\mathrm{CP}$-violation may be restricted to the phase transition itself, a phenomenon which is called "transitional CP-violation". In particular, this scenario allows for a large amount of CP-violation during the process of baryon production, while being completely unconstrained by experiment.

To start with, let us summarize the case of CP-violation at zero temperature. In the MSSM the tree-level Higgs potential automatically conserves CP. However, explicit CPviolation emerges from possible complex phases of the soft SUSY breaking $A$-terms and gaugino masses, and from the $\mu$-parameter [4]. These phases contribute to the electric dipole moments (EDMs) of quarks and electrons [38]. From the experimental upper limits [39] on the neutron EDM, $d_{n}<1.1 \times 10^{-25} e \mathrm{~cm}$, and the electron EDM, $d_{e}<4.3 \times 10^{-27} e$ $\mathrm{cm}$, constraints on the supersymmetric phases can be derived. Conservatively, rather small supersymmetric phases of the order $\mathcal{O}\left(10^{-2}-10^{-3}\right)$ are required to satisfy the experimental bounds [38]. Larger phases may only be tolerated if the first and second generation squarks have masses in the $\mathrm{TeV}$ range [40], or if accidental cancellations do occur. Recently it was realized that these cancellations are more generic than thought previously 41]. Explicit CP-violation occurring in the SUSY breaking sector of the MSSM is communicated to the Higgs sector by radiative corrections. However, the complex phases induced in the Higgs vevs are much too small to have any phenomenological implications [42]. Nevertheless, Higgs phenomenology may be significantly changed due to mixing of the CP-even and CP-odd Higgs states induced by complex mass parameters and coupling constants [4].

The Higgs sector of the $Z_{3}$-symmetric NMSSM contains one not removable phase which can be chosen to be the phase of $\lambda k^{*}$. This phase is not very much constrained provided $|\lambda|$ is significantly smaller than one [43]. If we allow for $Z_{3}$-symmetry breaking, six possibly complex parameters appear in the tree-level Higgs potential.] Two phases can be absorbed by a redefinition of the Higgs and singlet fields. Thus we can take $\mu B+\lambda r^{*}$ and $k r^{*}$ being real valued, without loss of generality. We parametrize the Higgs and singlet fields according to

$$
H_{1}^{0}=\bar{h}_{1} e^{i \theta_{1}}, \quad H_{2}^{0}=\bar{h}_{2} e^{i \theta_{2}}, \quad S=\bar{s} e^{i \theta_{S}},
$$

and define $\mu=\bar{\mu} e^{i \phi_{\mu}}, \lambda=\bar{\lambda} e^{i \phi_{\lambda}}$, etc. Furthermore, we introduce

$$
\theta=\theta_{1}+\theta_{2}, \quad \bar{\theta}=\theta_{1}-\theta_{2} .
$$

\footnotetext{
${ }^{7}$ In this section we relax the assumption of universal soft SUSY breaking which would induce correlations between the various phases at the weak scale.
} 
Because of gauge invariance the Higgs potential is independent of the phase combination $\bar{\theta}$. Using these definitions the tree-level Higgs potential takes the form

$$
\begin{aligned}
V_{\text {tree }}= & \left(\bar{\mu}^{2}+\bar{\lambda}^{2} \bar{s}^{2}+2 \bar{\lambda} \bar{\mu} \bar{s} \cos \left(\theta_{S}+\phi_{\lambda}-\phi_{\mu}\right)\right)\left(\bar{h}_{1}^{2}+\bar{h}_{2}^{2}\right)+\bar{\lambda}^{2} \bar{h}_{1}^{2} \bar{h}_{2}^{2}+\bar{k}^{2} \bar{s}^{4} \\
& +2 \bar{\lambda} \bar{k} \bar{s}^{2} \bar{h}_{1} \bar{h}_{2} \cos \left(\theta-2 \theta_{S}+\phi_{\lambda}-\phi_{k}\right)+\frac{g_{1}^{2}+g_{2}^{2}}{8}\left(\bar{h}_{1}^{2}-\bar{h}_{2}^{2}\right)^{2} \\
& +2\left(\lambda r^{*}+\mu B\right) \bar{h}_{1} \bar{h}_{2} \cos \theta+2 k r^{*} \bar{s}^{2} \cos \left(2 \theta_{S}\right)+m_{H_{1}}^{2} \bar{h}_{1}^{2}+m_{H_{2}}^{2} \bar{h}_{2}^{2}+m_{S}^{2} \bar{s}^{2} \\
& +2 \bar{\lambda} \bar{A}_{\lambda} \bar{s}_{1} \bar{h}_{2} \cos \left(\theta+\theta_{S}+\phi_{\lambda}+\phi_{A_{\lambda}}\right)+\frac{2}{3} \bar{k}_{k} \bar{A}_{k} \bar{s}^{3} \cos \left(3 \theta_{S}+\phi_{k}+\phi_{A_{k}}\right) .
\end{aligned}
$$

Note that in contrast to the MSSM the phase of the $\mu$-parameter enters $V_{\text {tree }}$. Explicit CP-violation automatically generates complex vevs for the Higgs and singlet fields. In the general NMSSM this is a tree-level effect which should be taken into account. An example will be discussed below. EDM constraints on CP-violating phases in the general NMSSM depend on the coupling of these phases to the (MS)SM sector. Phases which directly enter the squark, chargino and neutralino mass matrices, e.g. $\phi_{\mu}$ and $\langle\theta\rangle$, have to obey the MSSM bounds which have been discussed above. The other phases, e.g. $\phi_{k}$ and $\left\langle\theta_{S}\right\rangle$, are less constrained, provided the coupling to the MSSM sector, $\lambda$, is sufficiently small 443 .

Even when the Lagrangian is CP-conserving, the CP-symmetry may be spontaneously violated by complex scalar vevs, i.e. $\mathrm{CP}$ and the $S U(2) \times U(1)$ gauge symmetry are broken together at the EWPT. In the MSSM radiative corrections to the Higgs potential can induce a CP-violating vacuum. However, the required Higgs boson with mass of a few $\mathrm{GeV}$ is obviously ruled out by experiment [42]. The $Z_{3}$-symmetric NMSSM provides only limited improvement on the minimal model: radiative corrections generate spontaneous CP-violation, provided the lightest neutral Higgs mass is smaller than about $40 \mathrm{GeV}$ 44]. In the general NMSSM the situation is very different. Spontaneous CP-violation (SCPV) is possible even at tree-level. However, Higgs spectra consistent with the experimental Higgs mass bounds require nearly maximal CP-violation, i.e. $\langle\theta\rangle,\left\langle\theta_{S}\right\rangle$ of the order $\mathcal{O}(1)$ 45. Phases $\theta \sim 0.1$ are only possible for Higgs masses smaller than $30 \mathrm{GeV}$. This finding is related to the fact that $\mathrm{CP}$ is a discrete symmetry. If $\mathrm{CP}$ is spontaneously broken there arise two degenerate minima $\vartheta^{\beta}$ with phases $\pm\langle\theta\rangle$, which are nearby when $\langle\theta\rangle$ becomes small. From the SM Higgs potential it is known that the squared mass of the Higgs boson is proportional to $\lambda^{\prime}\langle h\rangle^{2}$, where $\lambda^{\prime}$ denotes the quartic coupling. In the case of small SCPV we can identify $\lambda^{\prime}\langle h\rangle^{2} \rightarrow \lambda^{\prime} v^{2}\langle\theta\rangle^{2}$, which up to numerical prefactors of order unity is the mass of the emerging light, almost CP-odd Higgs boson. Obviously, for small $\langle\theta\rangle$ this state becomes arbitrarily light.

Thus SCPV in the NMSSM does not appear to be very promising. Small phases are ruled out because they require a light Higgs boson, while large phases are tightly constrained by the EDM experiments. We will see below that this conclusion does not apply to SCPV which is only present during the PT.

We now derive the equations of motion for moduli and phases of the Higgs fields.? Using the definitions (5.14) and (5.15) we can rewrite the kinetic terms for the Higgs

\footnotetext{
${ }^{8}$ For that reason explicit CP-violation has also to be present in the model in order to avoid domain walls when CP is broken at the EWPT. However, this explicit phase may be much smaller than one.

${ }^{9}$ The singlet field is most conveniently split into real and imaginary part which can be treated along the lines discussed in the previous section.
} 
bosons in the Lagrangian according to

$$
\mathcal{L}=\partial_{\mu} \bar{h}_{1} \partial^{\mu} \bar{h}_{1}+\partial_{\mu} \bar{h}_{2} \partial^{\mu} \bar{h}_{2}+\frac{\bar{h}_{1}^{2}+\bar{h}_{2}^{2}}{4}\left(\partial_{\mu} \theta \partial^{\mu} \theta+\partial_{\mu} \bar{\theta} \partial^{\mu} \bar{\theta}\right)+\frac{\bar{h}_{1}^{2}-\bar{h}_{2}^{2}}{2} \partial_{\mu} \theta \partial^{\mu} \bar{\theta}+\ldots
$$

Since $\partial_{\bar{\theta}} V_{T}=0$ the Euler-Lagrange equation for $\bar{\theta}$ implies

$$
\left(\bar{h}_{1}^{2}+\bar{h}_{2}^{2}\right) \partial^{\mu} \bar{\theta}+\left(\bar{h}_{1}^{2}-\bar{h}_{2}^{2}\right) \partial^{\mu} \theta=c_{\theta}^{\mu}=\text { const. }
$$

In general, the integration constant $c_{\theta}^{\mu}$ cannot be set to zero without introducing a pure gauge field. However, a non-vanishing $c_{\theta}^{\mu}$ would enhance the energy of a bubble configuration, so we will set $c_{\theta}^{\mu}=0$ in the following. After elimination of $\partial_{\mu} \bar{\theta}$ by help of (5.18) the equations of motion for $\bar{h}_{1}, \bar{h}_{2}$ and $\theta$ take the form

$$
\begin{aligned}
2 \frac{d^{2}}{d z^{2}} \bar{h}_{1}+\frac{2 \bar{h}_{1} \bar{h}_{2}^{4}}{\left(\bar{h}_{1}^{2}+\bar{h}_{2}^{2}\right)^{2}} \frac{d^{2}}{d z^{2}} \theta-\frac{\partial}{\partial \bar{h}_{1}} V_{T}=0 \\
2 \frac{d^{2}}{d z^{2}} \bar{h}_{2}+\frac{2 \bar{h}_{2} \bar{h}_{1}^{4}}{\left(\bar{h}_{1}^{2}+\bar{h}_{2}^{2}\right)^{2}} \frac{d^{2}}{d z^{2}} \theta-\frac{\partial}{\partial \bar{h}_{2}} V_{T}=0 \\
\frac{d}{d z}\left[\frac{2 \bar{h}_{1}^{2} \bar{h}_{2}^{2}}{\bar{h}_{1}^{2}+\bar{h}_{2}^{2}} \frac{d}{d z} \theta\right]-\frac{\partial}{\partial \theta} V_{T}=0
\end{aligned}
$$

where we restricted ourselves to the case of the static domain wall perpendicular to the $z$-direction (4.11). The equations for the critical bubble follow along the same lines. In eqs. (5.19) - (5.21) the phase $\theta$ is dynamical only if both moduli, $\bar{h}_{1}$ and $\bar{h}_{2}$, are different from zero. This demonstrates that in the bubble wall $\theta$ cannot be assigned to one of the Higgs fields.

In the previous section we noted that $\tan \beta$ varies only slightly in the bubble wall. Neglecting this small perturbation the equations (5.19) - (5.21) can be further reduced and we obtain for the variation of the phases of the Higgs fields $H_{1}^{0}$ and $H_{2}^{0}$

$$
\delta \theta_{1}=\sin ^{2}\left(\beta_{T}\right) \delta \theta, \quad \delta \theta_{2}=\cos ^{2}\left(\beta_{T}\right) \delta \theta .
$$

Here $\tan \beta_{T}$ represents the ratio of the Higgs field moduli in the broken minimum at the critical temperature. In the limit of large $\tan \left(\beta_{T}\right)$ the variation of $\theta$ is almost completely absorbed by $\theta_{1}$, while $\theta_{2}$ remains more or less constant. For example, in case of $\tan \beta_{T}=5$ we obtain $\delta \theta_{2}=0.038 \delta \theta$, which is small even for $\delta \theta=\mathcal{O}(1)$. In section 6 we will discuss that the variations of complex phases fuel baryon production, rather than the complex phases themselves. As a consequence, baryon number generation resulting from a varying phase in the top quark mass, $m_{t}=y_{t} \sin \left(\beta_{T}\right) \bar{h} e^{i \theta_{2}}$, will turn out to be highly suppressed in the limit of large $\tan \left(\beta_{T}\right)$. Since in the region of the parameter space considered in this work the Higgs vev ratio changes only by a few percent when $T$ is raised from zero to the critical temperature, this is the case in the regime of large $\tan \beta$.

We now discuss the implications of the general equations of motion derived above. Let us start with explicit CP-violation. In the MSSM the complex phases which are induced in the Higgs vevs by CP-violating couplings are completely negligible for realistic Higgs masses. In ref. [5] we found this behavior confirmed at finite temperature: $\theta \lesssim \mathcal{O}\left(10^{-3}\right)$, even for explicit phases of the order of one. The variation of $\theta$ in the bubble is of the 

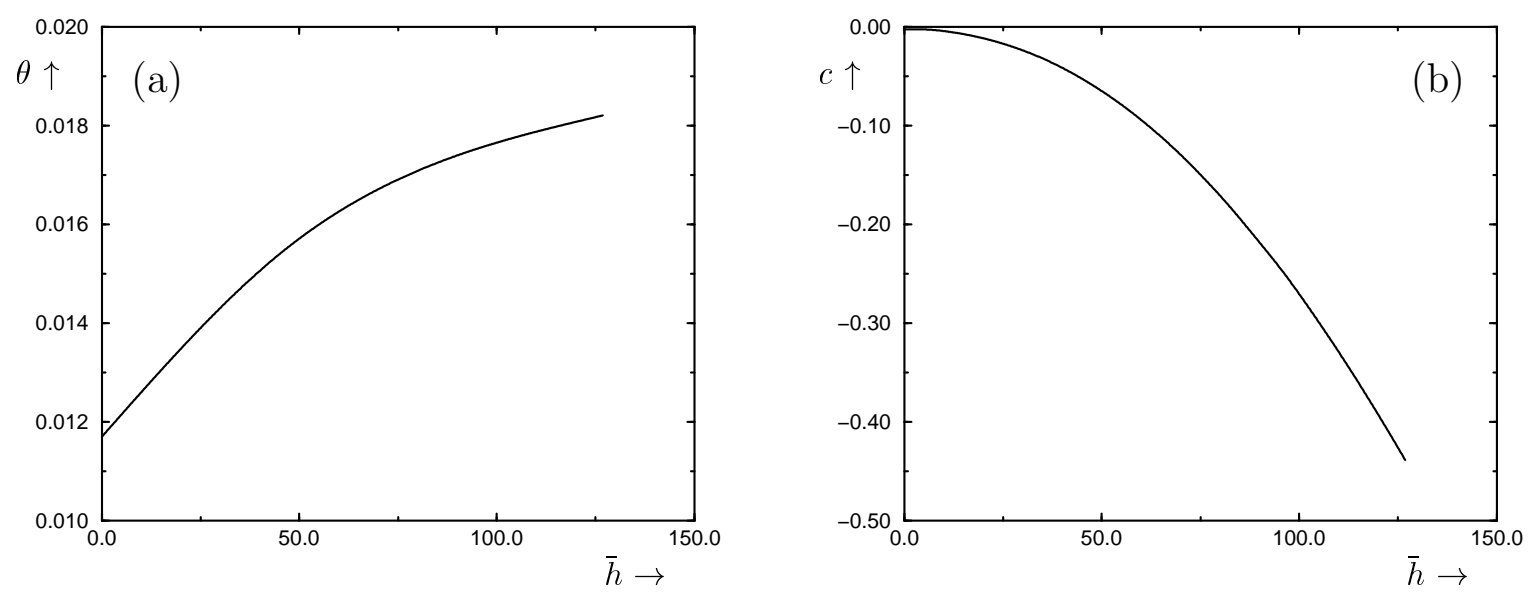

Figure 6: (a): Variation of the CP-violating fields $\theta$ (a) and $c(\mathrm{~b})$ in the bubble wall as a function of $\bar{h}=\sqrt{\bar{h}_{1}^{2}+\bar{h}_{2}^{2}}$ at the critical temperature for $\phi_{\mu}=0.1, A_{0}=-100 \mathrm{GeV}$ and $M_{0}=125 \mathrm{GeV}$. The remaining parameters are chosen as in fig. 20a. (Units in $\mathrm{GeV}$.)

same order of magnitude. We conclude that in the MSSM with explicit CP-violation, the Higgs vevs can be taken real to very good approximation.

In the NMSSM the situation is different, because explicit CP-violation is possible in the tree-level Higgs potential (5.16). In general, the behavior of the complex valued Higgs and singlet fields in the bubble wall can only be reliably determined by using the minimization techniques of ref. [33]. In order to make this possible, the equations of motion which describe $\mathrm{s}=\operatorname{Re}(S), \mathrm{c}=\operatorname{Im}(S), \bar{h}_{1}, \bar{h}_{2}$ and $\theta$ have to be included in the $\mathcal{F}$. In ref. [5] this approach was applied to compute $\mathrm{CP}$-violating bubble wall profiles in the MSSM and NMSSM. It turned out that also the phase $\theta$ is rather accurately described by a kink-ansatz (4.12). Moreover, it was verified that in the calculation of the wall shape small CP-violating phases $\theta \lesssim \mathcal{O}\left(10^{-1}\right)$ can indeed be treated as perturbations of a CPconserving solution. In this case the bubble wall profile can be conveniently determined in two steps: first, one computes the CP-conserving profile of the fields $\bar{h}_{1}, \bar{h}_{2}$ and $s$, where the CP-violating fields $\theta$ and $c$ are set to zero. In a second step, the CP-violating constituents of the bubble wall may be computed by simply minimizing the effective potential $V_{T}\left(\bar{h}_{1}, \bar{h}_{2}, \theta, s, c\right)$ with respect to $\theta$ and $c$, while keeping $\bar{h}_{1}, \bar{h}_{2}$ and $s$ fixed. The variation of the Higgs vev ratio can be neglected in this calculation. We emphasize that this prescription works only in case of small CP-violation in the Higgs and singlet fields.

If $V_{T}\left(\bar{h}_{1}, \bar{h}_{2}, \theta=0, s, c=0\right)$ has a smooth ridge, the profile of the CP-conserving fields can be obtained from the improved shooting method discussed in section 4 . Hence the complete CP-violating bubble profile can be computed without minimizing the functional $\mathcal{F}$.

In fig. 6 we display an example of a bubble wall in presence of explicit CP-violation using $\phi_{\mu}=0.1, A_{0}=-100 \mathrm{GeV}$ and $M_{0}=125 \mathrm{GeV}$, the remaining parameters are chosen as in fig. 2a. The lightest Higgs boson has a mass $M_{h}=86 \mathrm{GeV}$. We find $T_{c}=101$ $\mathrm{GeV}$ and $v_{c} / T_{c}=1.77$, so the $\mathrm{PT}$ is strongly first order. For the wall thickness we obtain $L_{w}=5 / T$. The CP-even singlet field evolves from $s=115 \mathrm{GeV}$ in the symmetric phase to $s=-79 \mathrm{GeV}$ in the Higgs phase. From fig. 6a we take $\theta=0.0182$ in the broken phase, and $\delta \theta=0.0065$. In the MSSM, $\phi_{\mu} \sim 1$ would be required to induce an effect of the same 


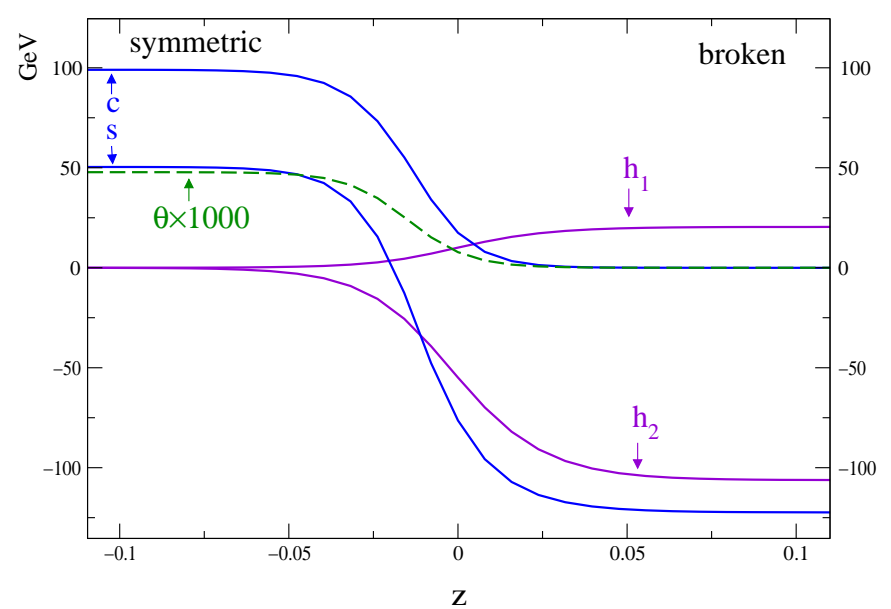

Figure 7: Transitionally CP-violating bubble wall profile from the parameter set of table 1. The position variable $z$ is given in units of $\mathrm{GeV}^{-1}$.

magnitude. Also the CP-odd component of the singlet field, $c$, varies in the bubble wall, as is shown in fig. 6 $6 \mathrm{~b}$. In the symmetric phase we have $c=0$, since $\phi_{\mu}$ couples to the singlet only in case of non-vanishing $\bar{h}_{1,2}$.

We now turn to the case of spontaneous CP-violation. Although this scenario is disfavored at zero temperature, the Higgs and singlet fields may still acquire large complex phases at high temperatures, or even only during the EWPT. Since it will turn out in section 6 that explicit CP-violation can account for the observed baryon asymmetry only in special regions of the NMSSM parameter space, spontaneous CP-violation at finite temperature becomes an interesting alternative scenario.

In the $Z_{3}$-symmetric NMSSM spontaneous CP-violation at finite temperature has already been investigated some years ago in ref. [46], where an effective potential truncated at the renormalizable level was used. It was found that spontaneous CP-violation cannot occur in the finite temperature broken phase while having a viable temperature zero phenomenology, since thermal corrections to the effective potential have the tendency to restore symmetries. The authors suggested that spontaneous CP-violation may take place during the EWPT ("transitional CP-violation"), i.e. the Higgs and singlet fields may acquire complex phases in the bubble wall, although no definite example was given. However, the truncated effective potential used in their analysis proved to give misleading results in case of the MSSM [5], as will be discussed below.

In our analysis of finite temperature spontaneous CP-violation in the general NMSSM we use the full 1-loop effective potential (3.8) without any truncations. It turns out that spontaneous CP-violation does not occur for the universal pattern of SUSY breaking discussed in section 2, which induces too large masses for the CP-odd Higgs bosons. However, spontaneous CP-violation can take place if universality is violated in the singlet sector. In

\begin{tabular}{|c|c|c|c|c|c|c||c|c|c|}
\hline$x$ & $\tan \beta$ & $\lambda$ & $k$ & $M_{0}$ & $A_{0}$ & $m_{0}$ & $A_{\lambda}$ & $A_{k}$ & $m_{S}^{2}$ \\
\hline-150 & -5 & 0.05 & 0.4 & 100 & -100 & 200 & -150 & 50 & -2000 \\
\hline
\end{tabular}

Table 1: Parameter set used in fig. 7 (all dimensionful parameters in GeV). 
this case $A_{\lambda}, A_{k}$ and $m_{S}^{2}$ are free parameters. 四 Fig. 0, which is taken from ref. [5], shows an example of a transitionally CP-violating bubble wall for the parameters given in table 1, which was computed using the minimization algorithm of ref. [33]. More precisely, the scenario corresponds to a phase transition between an electroweak symmetric high temperature phase, where $\mathrm{CP}$ is violated by a complex singlet vev, and an electroweak broken, CP-conserving low temperature phase, i.e. $(s, c)_{\text {sym }}=(50 \mathrm{GeV}, 99 \mathrm{GeV}) \rightarrow(s, c)_{\text {brk }}=$ $(-122 \mathrm{GeV}, 0)$. The critical temperature is found to be $101 \mathrm{GeV}$, and we have a strong first order PT with $v_{c} / T_{c}=1.6$. The mass of the lightest CP-even Higgs boson is $84 \mathrm{GeV}$ which because of its reduced coupling to the Z-boson is still compatible with the experimental data. There is also a rather light CP-odd state with a mass of $105 \mathrm{GeV}$, which is almost a pure singlet. The bubble wall is found to be rather thin with $L_{w} \sim 3 / T$. In our example spontaneous CP-violation is triggered in the singlet sector. The small coupling $\lambda$ communicates it to the Higgs fields. As a consequence, the almost maximal phase of the singlet field, $\theta_{s}$, induces only a small phase $\theta \sim 1 / 20 \sim \lambda \theta_{s}$ in the Higgs sector. Up to now we only identified one particular region in the NMSSM parameter space where transitional CP-violation occurs. A more systematic study would be very desirable. Especially it would be interesting to find an example where transitional CP-violation originates from the Higgs fields rather than the singlet field. In that case $\theta$ is expected to be of the order one.

Let us finally comment on transitional CP-violation in the MSSM, where there also have been claims in the literature that transitional may occur [47. In ref. 48] even an example of a transitionally CP-violating parameter set has been presented. All these studies were carried out using an effective potential that has been truncated at the renormalizable level, i.e. the logarithmic terms, etc., were neglected. In ref. [5] we performed a systematic search for transitional CP-violation in the MSSM using the full 1-loop thermal effective potential without finding any viable parameter set. In particular, the example of transitional CP-violation presented in ref. 48 could not be confirmed. This discrepancy appears to be due to the different approximations which have been made concerning the effective potential. Our results indicate that the truncated effective potential is not appropriate to discuss transitional CP-violation.

\section{Baryon Asymmetry in the Semi-Classical Limit}

\subsection{Generalities}

Between the initial nucleation and the completion of the phase transition expanding bubbles convert the symmetric phase into the broken phase. The early universe is filled with a hot plasma of particles, most of them have rather different masses and mixings inside and outside the bubble. Thus the bubble wall behaves like a potential on which the particles scatter. At the bubble surfaces the plasma is thrown out of equilibrium by the motion of the phase boundary. The higher pressure inside the bubble tends to accelerate the wall, while the interaction between the wall and the particles in the plasma dissipates energy and slows the wall down. Finally, a stationary situation is reached, where the different forces balance and the wall propagates with constant velocity $v_{w}$. In general, the wall velocity depends on the shape of the effective potential and on the composition of the

\footnotetext{
${ }^{10}$ It is even sufficient to violate universality only via $m_{S}^{2}$.
} 
plasma.

In the symmetric phase baryon number violation occurs frequently due to hot sphaleron processes. A baryon asymmetry is produced, if the departure from equilibrium at the phase boundary biases the baryon number violating processes in a CP-violating fashion. The most efficient mechanisms of baryon number generation rely on transport. If CP is violated in the interaction between the bubble wall and the particles in the plasma, different population densities for particles and anti-particles are induced in front of the wall. The difference between the particle and anti-particle populations is then transported into the symmetric phase, where it biases rapid baryon number violation. The total amount of baryon number which gets produced during the phase transition depends crucially on the shape and motion of the bubble wall.

Different methods have been suggested in the literature in order to describe the effects of CP-violating interactions between particles in the plasma and the propagating bubble wall, which finally generate CP-violating source terms that enter the diffusion equations for particle transport. Depending on the properties of the bubble wall, in the first place its velocity $v_{w}$ and thickness $L_{w}$, different approximations may be applied. The most rigorous approach is based on the closed time-path (CTP) formulation of non-equilibrium quantum field theory [50]. It leads to a set of quantum Boltzmann equations [51] which describe the temporal evolution of particle densities including particle number changing interactions and CP-violating source terms [52]. However, the approximations and results are still controversely discussed. There have also been efforts to use the CTP formalism only in calculating the $\mathrm{CP}$-violating source terms which in turn are inserted into classical Boltzmann equations 49.

If the thickness of the bubble wall $L_{w}$ is smaller than the mean free path $l$ of the particle under consideration ("thin wall"), one may neglect the influence of the plasma during the scattering of the particle off the wall. The interaction of a fermion or boson with the wall can then approximately be described by using a "free" Dirac or Klein-Gordon equation, respectively. CP-violation is encoded in different reflection and transmission coefficients for particles and anti-particles [53 [57]. In ref. [58] also the effects of thermal scattering were taken into account, which due to decoherence have a negative impact on the generation of a CP-violating observable [59]. Baryon production is fueled by a net flux of charge into the symmetric phase ("charge transport mechanism" [53]), which is induced by the reflection asymmetry. This current is subsequently inserted into a set of classical Boltzmann equations which determines the evolution of the particle distributions. Recently, reflection and transmission probabilities have also been calculated by using standard quantum field theory methods [60]. There, thermal scattering, i.e. damping, was included via an imaginary part of the self-energy.

As the bubble wall becomes thicker, $L_{w} \sim l$, interactions with the plasma must inevitably be taken into account, when a particle encounters the propagating wall. In general, this requires non-equilibrium quantum field theory methods [52]. However, if $L_{w} \gg 1 / T$, most particles have inverse momenta $1 / p \ll L_{w}$ and may therefore be treated semi-classically 61 64]. Using the WKB approximation one can derive dispersion relations which in the presence of CP-violation are different for particles and anti-particles. The dispersion relations then enter classical Boltzmann equations, leading to a unified description of CP-violating source terms, particle scattering and transport. Recently, it was clarified how the semi-classical description arises from the quantum Boltzmann equations in the limit of a slowly varying background field (thick wall regime) [65]. Of course, 
even in the thin wall limit the high-momentum particles may be described semi-classically. However, in that case they only give a sub-leading contribution to the CP-violating source, which in this case is dominated by the low-momentum particles. Even in case of thick walls there may be important contributions from low-momentum modes neglected in the semi-classical treatment, which according to ref. [52 significantly enhance the produced amount of baryon number. There is also a very recent calculation [66] showing that gauge fields in the hot phase lower significantly the wall velocity, such also leading to a more efficient baryon production. In ref. [67 the semi-classical approximation to particle dynamics has been applied to calculate the bubble wall velocity in the SM. In ref. 668 it was shown that the stops in the MSSM considerably lower the wall velocity.

Notice that different particle species with different (gauge) interaction have different mean free paths. Thus whether one is in the thin or thick wall regime depends on the particle species under consideration. In the (N)MSSM especially particles with strong interactions (quarks and squarks) require a thick wall treatment.

There is some disagreement among the groups that have estimated the baryon asymmetry generated during the EWPT in the MSSM. In refs. [49, 52, 58] the CP-violating source terms are proportional to the variation of $\tan \beta$ in the wall, which according to the discussion of section 4 causes a suppression of at least $\mathcal{O}\left(10^{-2}\right)$. This dependence is due to taking into account only the leading order of the Higgs insertion expansion, which has been used to calculate source terms. At higher orders in the expansion there arise contributions, which escape the $\delta \beta$ suppression, as was recently shown in ref. 60. However, these corrections turn out very small and are competitive to the leading order contributions only in case of $\delta \beta<\mathcal{O}\left(10^{-3}\right)$. In the recent paper $[71]$ it was argued convincingly that the $(\tan \beta)^{\prime}$ source cancels and that one has to consider closely a source term symmetrical in the two Higgs fields.

In this section we generalize the method introduced in ref. 64 to calculate the baryon asymmetry in the NMSSM, which has not been considered so far. Different from ref. [64] our formulas also cover the case of CP-violating bubble walls. We obtain a non-vanishing baryon asymmetry even in case of constant $\tan \beta$, which is due to the variation of the singlet field or the presence of CP-violating bubble walls.

\subsection{WKB approximation and dispersion relations}

The idea of deriving CP-violating source terms using a semi-classical approximation was developed in context of the two Higgs doublet model [63] and afterwards applied to the charginos in the MSSM [64]. In the following we review the derivation of semi-classical dispersion relations and generalize this method to account for the effects of CP-violating bubble walls, which potentially are present in the NMSSM. These additional sources of CP-violation will turn out to be very helpful in order to generate a baryon to entropy ratio in the observed range: $\eta_{B}=n_{B} / s \sim 2-7 \times 10^{-11}$ [69]. We also give the dispersion relations in the bosonic case. In the next section these dispersion relations enter the classical Boltzmann equations that describe particle transport induced by the propagating bubble wall. 


\subsubsection{The fermionic case}

We start the derivation of fermionic dispersion relations in presence of CP-violation with a simple example: A single (Dirac-) fermion $\Psi_{D}^{T}=\left(\eta_{\alpha}, \bar{\chi}^{\dot{\alpha}}\right)$ that couples only to one of the two Higgs doublets, which we denote by $H$. The most prominent realizations are the quarks and leptons of the (N)MSSM in the presence of a CP-violating bubble wall. Due to its coupling to the Higgs, $y$, the fermion obtains a mass proportional to the Higgs vev, $M=y H$. During the passage of the bubble wall the fermion mass becomes space-time dependent. Assuming that the bubble has grown to macroscopic size and reached its final velocity, we can neglect the curvature of the wall and boost to the rest frame of the bubble. Then the fermion mass only depends on one position coordinate, which we denote by $z$, i.e. $M=M(z)$. As a consequence, the energy of the particle, $E$, and its momentum perpendicular to the $z$-direction, $p_{\perp}$, are constants of motion. Writing $M=m e^{i \theta}$, possible CP-violation is encoded in a non-vanishing phase $\theta$.⿴囗十 Any constant $\theta$ can be absorbed by a redefinition of the fermion field. When couplings to additional fields are neglected only a varying phase contributes to CP-violation.

In the presence of a complex mass term the fermion field is described by the free Dirac equation

$$
\left(i \gamma^{\mu} \partial_{\mu}-P_{L} M-P_{R} M^{*}\right) \Psi_{D} \equiv\left(\begin{array}{cc}
-M & i \sigma^{\mu} \partial_{\mu} \\
i \bar{\sigma}^{\mu} \partial_{\mu} & -M^{*}
\end{array}\right)\left(\begin{array}{l}
\eta \\
\bar{\chi}
\end{array}\right)=0
$$

Notice that at this level all interactions between the particle and the plasma are neglected. The scattering effects will be accounted for in the next section when Boltzmann equations are written down that describe the local phase space distributions. We are working in the chiral representation of the $\gamma$-matrices. Exploiting conservation of energy and boosting to the Lorentz frame where $p_{\perp}=0$ we can take the ansatz $\Psi_{D}=e^{-i E t} \xi(z)$ and are left with a one dimensional problem. The interaction between the fermion and the wall conserves the $z$-component of the spin, $S_{z}$. Thus eq. (6.23) splits into two equations 55.

$$
i \partial_{z} \xi_{ \pm}= \pm Q(z) \xi_{ \pm}, \quad Q(z)=\left(\begin{array}{cc}
E & -m(z) e^{-i \theta(z)} \\
m(z) e^{i \theta(z)} & -E
\end{array}\right)
$$

where $\xi_{+}=\left(\xi_{1}, \xi_{3}\right)$ and $\xi_{-}=\left(\xi_{2}, \xi_{4}\right)$ are the $S_{z}= \pm \frac{1}{2}$ components of $\xi$. To solve eq. 6.24) one brings the $z$-dependent matrix $Q(z)=D(z) Q_{D}(z) D(z)^{-1}$ into a diagonal form, where 64

$$
Q_{D}=\left(\begin{array}{cc}
\sqrt{E^{2}-m^{2}} & 0 \\
0 & -\sqrt{E^{2}-m^{2}}
\end{array}\right), D=\left(\begin{array}{cc}
\cosh X & e^{-i \theta} \sinh X \\
e^{i \theta} \sinh X & \cosh X
\end{array}\right)
$$

and $\tanh 2 X=m / E$. In the local helicity basis, $\tilde{\xi}_{ \pm}=D^{-1} \xi_{ \pm}$, the Dirac equation 6.24) takes the form

$$
i \hbar \partial_{z} \tilde{\xi}_{ \pm}=\left( \pm Q_{D}-D^{-1} i \hbar \partial_{z} D\right) \tilde{\xi}_{ \pm}
$$

which still is an exact equation. In general, the correction term $D^{-1} i \hbar \partial_{z} D$ caused by the position dependent field redefinition is not of diagonal form. The two components

\footnotetext{
${ }^{11}$ Here $\theta$ denotes the phase of the Higgs boson the fermion is coupling to. It should not be mixed up with the common phase of both Higgs fields defined in eq. (5.15) which is denoted by the same symbol.
} 
of $\tilde{\xi}_{ \pm}$are still coupled. However, the off-diagonal part is proportional to $\partial_{z} D \sim D / L_{w}$. Typical momenta of the particles in the plasma are of the order of the temperature $T$, which is much larger than $1 / L_{w}$ for the bubbles under consideration. We therefore expand eq. (6.26) in powers of $\partial_{z}$ or more precisely in powers of $\hbar$ (WKB approximation) that we already reintroduced for that reason.

To order $(\hbar)^{0}$ we can neglect the $D^{-1} \hbar i \partial_{z} D$ contribution. Thus the two components of $\tilde{\xi}_{ \pm}$decouple in eq. (6.26). Inserting the WKB ansatz for the fermion field

$$
\tilde{\xi}_{ \pm}^{(1)}=\left(\begin{array}{l}
1 \\
0
\end{array}\right) e^{-\frac{i}{\hbar} \int^{z} p_{z}\left(z^{\prime}\right) d z^{\prime}}, \quad \tilde{\xi}_{ \pm}^{(2)}=\left(\begin{array}{l}
0 \\
1
\end{array}\right) e^{-\frac{i}{\hbar} \int^{z} p_{z}\left(z^{\prime}\right) d z^{\prime}}
$$

into (6.26), we obtain the dispersion relations $p_{z}(E)$

$$
\begin{array}{ll}
\tilde{\xi}_{+}^{(1)}, \tilde{\xi}_{-}^{(2)}: & p_{z}=\sqrt{E^{2}-m^{2}}, \\
\tilde{\xi}_{+}^{(2)}, \tilde{\xi}_{-}^{(1)}: & p_{z}=-\sqrt{E^{2}-m^{2}} .
\end{array}
$$

The momenta $p_{z}$ are the eigenvalues of the matrix entering the RHS of eq. (6.26). The eigenfunctions may more transparently be labeled by the chirality states they correspond to in the limit $m \rightarrow 0$, e.g. $\tilde{\xi}_{+}^{(1)} \sim \xi_{1} \sim \eta_{1}$, etc. The dispersion relations may then be combined to $p_{z}=\operatorname{sgn}\left(p_{z}\right) \sqrt{E^{2}-m^{2}}$ which holds for left-handed particles $\eta$ and right-handed particles $\bar{\chi}$. Obviously, in the classical limit the $\theta$-dependence completely disappears, demonstrating that CP-violation is indeed a quantum-mechanical phenomenon.

To solve the Dirac equation (6.26) to order $\hbar$ we have to take into account the $D^{-1} \hbar i \partial_{z} D$ term which reintroduces a coupling between the two components of $\tilde{\xi}_{ \pm}$. The dispersion relations $p_{z}(E)$ are obtained from the eigenvalues of the matrix $\pm Q_{D}-D^{-1} i \hbar \partial_{z} D$. Since to order $\hbar$ the off-diagonal terms do not contribute, we are left with [64]

$$
\begin{aligned}
L(\eta): & p_{z}=\operatorname{sgn}\left(p_{z}\right) \sqrt{E^{2}-m^{2}}-\hbar \theta^{\prime} \sinh ^{2} X, \\
R(\bar{\chi}): & p_{z}=\operatorname{sgn}\left(p_{z}\right) \sqrt{E^{2}-m^{2}}+\hbar \theta^{\prime} \sinh ^{2} X,
\end{aligned}
$$

where $\theta^{\prime}=\partial_{z} \theta$ and

$$
\sinh ^{2} X=\frac{E-\sqrt{E^{2}-m^{2}}}{2 \sqrt{E^{2}-m^{2}}} .
$$

Again the states are labeled by their asymptotic chirality properties. Notice that the variation of $m$, which is encoded in $\partial_{z} X$, drops in the dispersion relations. The CP-violating part of the dispersion relation, $\Delta p_{z}=\hbar \theta^{\prime} \sinh ^{2} X$, is proportional to the derivative of the phase $\theta$. Thus only a varying phase contributes to CP-violation in the semi-classical limit. Furthermore, CP-violation is proportional to $\sinh ^{2} X$, which guarantees that its effect is turned off in the limit $m \rightarrow 0$, where $\theta$ is no longer well defined. Because of the different dispersion relations, left- and right-handed particles feel a different (semi-classical) force in their interaction with the wall. For the anti-particles, $\bar{\eta}(\bar{L})$ and $\chi(\bar{R})$, the CP-violating part comes with the opposite sign. Besides the force term there is a second manifestation of CP-violation: The phase $\theta$ enters also the transformation matrix to the helicity basis $D$. Since the interaction eigenstates are different from the helicity states, particles and anti-particles interact in a different way with the surrounding plasma. This generates a CP-violating source term which drives "spontaneous" baryogenesis [70. 
As pointed out recently in ref. [71], one should better use the kinetic momentum $p_{\text {kin }}=m v_{\text {group }}=m \frac{\partial E}{\partial p}$ instead of the canonical momentum $p$ (which we used up to now) in the quasi-classical limit of particles in the Boltzmann transport equations. This is quite in the spirit of the correspondence principle of basic quantum mechanics. Calculating the (inverse) group velocity from (6.29), and using (6.30), the kinetic moment beyond the zeroth order contains (order $\hbar$ ) correction terms

$$
\Delta p_{\text {kin }}= \pm \frac{\hbar \theta^{\prime} m^{2}}{2 E \sqrt{E^{2}-m^{2}}} .
$$

In the following we will need the dispersion relation for energy in terms of momentum which to order $\hbar$ is obtained as

$$
E_{ \pm} \equiv E_{0} \pm \Delta E=\sqrt{p_{\text {kin }}^{2}+m^{2}} \pm \operatorname{sign}\left(p_{z}\right) \theta^{\prime} \frac{m^{2}}{2\left(p_{\text {kin }}^{2}+m^{2}\right)}
$$

$E_{+}$is the energy of left-handed particles and anti-particles, whereas $E_{-}$corresponds to right-handed particles and anti-particles. ${ }^{\text {[z }}$ In the derivation of eq. (6.32) we transformed to a general Lorentz frame with non-zero momentum parallel to the wall. When boosting to the plasma frame the dispersion relation (6.32) is preserved to linear order in $v_{w}$ and $\theta^{\prime}$.

In our derivation of the dispersion relations we followed basically ref. [64]. CP-violation arises because of a position dependent phase in the transformation from the interaction (i.e. chirality) states to the local mass (i.e. helicity) eigenstates. In ref. 663 a slightly different approach was used to obtain the dispersion relation of a fermion (e.g. top quark) in the presence of a CP-violating Higgs field background, $H$, in context of the $2 \mathrm{HD}$ model: The complex phase in the fermion mass was removed by a gauge transformation, which induced a gauge field in the kinetic term of the fermion. The dispersion relation obtained with this technique is completely analogous to our result (6.29). However, it is not clear how to generalize this method to cover the case where several species mix, as occurs with the gauginos and Higgsinos in the (N)MSSM, or where the NMSSM singlet field background contributes to CP-violation. In both situations the CP-violating phase cannot be removed by a gauge transformation. On the other hand, the method described above is still applicable [64].

We are now in the position to address the problem of mixing Dirac fermions $\Psi_{D I}$, where the "flavor" index $I=1, \ldots, N$. In order to solve the corresponding Dirac equation we diagonalize the matrix

$$
Q(z)=\left(\begin{array}{cc}
\mathbf{1} E & -\mathbf{M}^{\dagger}(z) \\
\mathbf{M}(z) & -\mathbf{1} E
\end{array}\right)
$$

which is a straight forward generalization of (6.24). Here 1 denotes the unity matrix in flavor space and $\mathbf{M}$ is a position dependent complex mass matrix for the fermions, without further specifications. As a first step we write $\mathbf{M}$ and $\mathbf{M}^{\dagger}$ in terms of two unitary matrices $\mathbf{U}$ and $\mathbf{V}$, and a diagonal matrix $\mathbf{M}_{D}=\mathbf{m} e^{i \boldsymbol{\theta}}$

$$
\mathbf{M}=\mathbf{V M}_{D} \mathbf{U}^{\dagger}, \quad \mathbf{M}^{\dagger}=\mathbf{U M}_{D}^{\dagger} \mathbf{V}^{\dagger}
$$

\footnotetext{
${ }^{12}$ Notice that in ref. [64] the phase $\theta$ has been defined with the opposite sign.
} 
$\mathbf{U}$ and $\mathbf{V}$ may be obtained by diagonalizing the hermitian matrices $\mathbf{M}^{\dagger} \mathbf{M}$ and $\mathbf{M} \mathbf{M}^{\dagger}$, respectively. The transformation to the helicity basis takes the form

$$
\tilde{\boldsymbol{\xi}}_{ \pm}=\left(\begin{array}{cc}
\cosh \mathbf{X} & -e^{-i \boldsymbol{\theta}} \sinh \mathbf{X} \\
-e^{i \boldsymbol{\theta}} \sinh \mathbf{X} & \cosh \mathbf{X}
\end{array}\right)\left(\begin{array}{cc}
\mathbf{U}^{\dagger} & 0 \\
0 & \mathbf{V}^{\dagger}
\end{array}\right) \boldsymbol{\xi}_{ \pm} \equiv \mathbf{T}^{-1} \boldsymbol{\xi}_{ \pm}
$$

where $\mathbf{X}$ is a diagonal matrix in flavor space, obeying $\tanh (2 \mathbf{X})=\mathbf{m} / E$. Like in the single fermion case the CP-violating part of the dispersion relation is encoded in the diagonal elements of $\mathbf{T}^{-1} i \hbar \partial_{z} \mathbf{T}$. We find

$$
\begin{aligned}
& \tilde{\xi}_{ \pm}^{(1)}: p_{z}= \pm \sqrt{E^{2}-m_{I}^{2}}-\theta_{I}^{\prime} \sinh ^{2} X_{I}-\cosh ^{2} X_{I}\left[\mathbf{U}^{\dagger} i \partial_{z} \mathbf{U}\right]_{I}+\sinh ^{2} X_{I}\left[\mathbf{V}^{\dagger} i \partial_{z} \mathbf{V}\right]_{I} \\
& \tilde{\xi}_{ \pm}^{(2)}: p_{z}=\mp \sqrt{E^{2}-m_{I}^{2}}+\theta_{I}^{\prime} \sinh ^{2} X_{I}-\cosh ^{2} X_{I}\left[\mathbf{V}^{\dagger} i \partial_{z} \mathbf{V}\right]_{I}+\sinh ^{2} X_{I}\left[\mathbf{U}^{\dagger} i \partial_{z} \mathbf{U}\right]_{I}(6.3
\end{aligned}
$$

Here the subscript $I$ on the RHS denotes the Ith diagonal element of the corresponding matrix, i.e. $m_{I}=m_{I I}$, and we dropped the factor $\hbar$. In addition to the $\theta^{\prime}$ contribution, which we already encountered in the case of a single Dirac fermion, the dispersion relations receive corrections due to the position dependent rotations in flavor space, $\mathbf{U}$ and $\mathbf{V}$.

In eqs. (6.35) and (6.36) we allowed for complex phases in the diagonal matrix $\mathbf{M}_{D}$. Since $\mathbf{U}$ and $\mathbf{V}$ contain $2 N^{2}-2$ real parameters it would be sufficient to take real values in $\mathbf{M}_{D}$ in order to reproduce the $2 N^{2}$ real parameters of $\mathbf{M}$. However, we have to omit the $N-1$ rotations in $\mathbf{U}$ and $\mathbf{V}$ which belong to the Abelian subgroup of $S U(N)$. These are not related to $\mathrm{CP}$-violation, but rather correspond to artificial redefinitions of the interaction (chirality) states, as can be deduced from the case vanishing fermion mixing. Thus allowing for complex values for the masses in $\mathbf{M}_{D}$ we end up with a one by one correspondence between the $N^{2}$ parameters in $\mathbf{M}$ and the ones contained in $\mathbf{U}, \mathbf{V}$ and $\mathbf{M}_{D}$.

Having resolved the ambiguity in the definition of the helicity basis, it is possible to determine the matrices $\mathbf{U}, \mathbf{V}$ and $\mathbf{M}_{D}$ numerically. The dispersion relations are then easily obtained by evaluating the expressions (6.36). Here we will concentrate on the charged Winos and Higgsinos in the (N)MSSM. Since they mix via the $2 \times 2$ chargino mass matrix

$$
\mathcal{L}=\cdots+\left(i \tilde{W}^{-}, \tilde{h}_{1}^{-}\right)\left(\begin{array}{cc}
M_{2} & g_{2}\left(H_{2}^{0}\right)^{*} \\
g_{2}\left(H_{1}^{0}\right)^{*} & \mu+\lambda S
\end{array}\right)\left(\begin{array}{c}
i \tilde{W}^{+} \\
\tilde{h}_{2}^{+}
\end{array}\right)
$$

analytic formulas can be obtained. The charged Winos and Higgsinos can be combined to a Dirac spinor $\Psi_{D}=\left(\Psi_{L}, \Psi_{R}\right)^{T}$, where $\Psi_{L}=\left(\tilde{W}^{+}, \tilde{h}_{2}^{+}\right)^{T}$ and $\Psi_{R}=\left(\overline{\tilde{W}^{-}}, \overline{\tilde{h}_{1}^{-}}\right)^{T}$. We parametrize the $\mathrm{SU}(2)$ matrices $\mathbf{U}$ and $\mathbf{V}$ by

$$
\mathbf{U}=\left(\begin{array}{cc}
\cos a & e^{-i \gamma} \sin a \\
-e^{i \gamma} \sin a & \cos a
\end{array}\right), \quad \mathbf{V}=\left(\begin{array}{cc}
\cos b & e^{-i \delta} \sin b \\
-e^{i \delta} \sin b & \cos b
\end{array}\right)
$$

According to the discussion above we dismissed phases multiplying the cos-terms. The diagonal elements of $\mathbf{U}^{\dagger} i \partial_{z} \mathbf{U}$ which enter the dispersion relations (6.36) read

$$
\left[\mathbf{U}^{\dagger} i \partial_{z} \mathbf{U}\right]_{1}=-\left[\mathbf{U}^{\dagger} i \partial_{z} \mathbf{U}\right]_{2}=-\gamma^{\prime} \sin ^{2} a .
$$

We observe that only the derivative of the complex phase $\gamma$ contributes, whereas the derivative of $a$ drops. Similar relations hold for $\mathbf{V}^{\dagger} i \partial_{z} \mathbf{V}$. Inserting these expressions 
into eq. (6.36) we obtain the dispersion relations for left- and right-handed particles and anti-particles

$$
\begin{aligned}
& L_{I}: p_{z}=\operatorname{sgn}\left(p_{z}\right) \sqrt{E^{2}-m_{I}^{2}}-\left(\theta_{I}^{\prime}+\delta^{\prime} \sin ^{2} b\right) \sinh ^{2} X_{I}+\gamma^{\prime} \sin ^{2} a \cosh ^{2} X_{I}, \\
& \bar{L}_{I}: p_{z}=\operatorname{sgn}\left(p_{z}\right) \sqrt{E^{2}-m_{I}^{2}}+\left(\theta_{I}^{\prime}+\delta^{\prime} \sin ^{2} b\right) \sinh ^{2} X_{I}-\gamma^{\prime} \sin ^{2} a \cosh ^{2} X_{I}, \\
& R_{I}: p_{z}=\operatorname{sgn}\left(p_{z}\right) \sqrt{E^{2}-m_{I}^{2}}+\left(\theta_{I}^{\prime}-\gamma^{\prime} \sin ^{2} a\right) \sinh ^{2} X_{I}+\delta^{\prime} \sin ^{2} b \cosh ^{2} X_{I}, \\
& \bar{R}_{I}: p_{z}=\operatorname{sgn}\left(p_{z}\right) \sqrt{E^{2}-m_{I}^{2}}-\left(\theta_{I}^{\prime}-\gamma^{\prime} \sin ^{2} a\right) \sinh ^{2} X_{I}-\delta^{\prime} \sin ^{2} b \cosh ^{2} X_{I} .
\end{aligned}
$$

In the symmetric phase $L_{2}$ and $\bar{R}_{2}$ evolve to the left-handed Higgsinos states $\tilde{h}_{2}^{+}$and $\tilde{h}_{1}^{-}$, respectively. The flavor transformations $\mathbf{U}$ and $\mathbf{V}$ are related to the parameters of the chargino mass matrix (6.37):

$$
\begin{aligned}
& \sin ^{2} a=2|A|^{2} / \Lambda(\Lambda+\Delta) \text { with } \\
& A=g_{2}\left(\left(M_{2} H_{2}^{0}\right)^{*}+(\mu+\lambda S) H_{1}^{0}\right) \\
& \Delta=\left|M_{2}\right|^{2}-|\mu+\lambda S|^{2}+g_{2}^{2}\left(\left|H_{1}^{0}\right|^{2}-\left|H_{2}^{0}\right|^{2}\right) \\
& \Lambda=\left(\Delta^{2}+4|A|^{2}\right)^{1 / 2}
\end{aligned}
$$

and $\gamma=\arg A$. This gives

$$
\gamma^{\prime} \sin ^{2} a=2 \operatorname{Im}\left(A^{*} A^{\prime}\right) / \Lambda(\Lambda+\Delta)
$$

and there are similar relations for $\sin ^{2} b, \delta$ and $\delta^{\prime} \sin ^{2} b$ exchanging $a$ and $b, H_{1}^{0}$ and $H_{2}^{0}, \gamma$ and $-\delta$. The mass eigenvalues read (in non-symmetric notation)

$$
\begin{aligned}
& {\left[M_{D}\right]_{11}=M_{2} \frac{\cos a}{\cos b}-g_{2}\left(H_{2}^{0}\right)^{*} \frac{\sin a}{\cos b} e^{-i \gamma},} \\
& {\left[M_{D}\right]_{22}=(\mu+\lambda S) \frac{\cos a}{\cos b}+g_{2}\left(H_{1}^{0}\right)^{*} \frac{\sin a}{\cos b} e^{i \gamma} .}
\end{aligned}
$$

For $\lambda=0$ these expressions agree with the results of ref. [71]. In this case one is left with the MSSM, and the phases $\gamma$ and $\delta$ only vary due to a change in the Higgs vev ratio tan $\beta$ or because of transitional CP-violation in the bubble wall. The first contribution is highly suppressed, since the variation of $\beta$ is at most $\sim 10^{-2}$ for realistic Higgs masses [33, 335, while transitional CP-violation most probably does not occur at all in the MSSM [5]. On the other hand, the contribution to the chargino dispersion relations stemming from the variation of the complex phases in $\mathbf{M}_{D}$ requires only explicit CP-violating phases in $\mu$ or $M_{2}$. Eq. (6.42) demonstrates that even though the phases in the two terms entering $\left[M_{D}\right]_{11,22}$ are position independent, their contribution to the resulting phase varies due to the change in the (real) Higgs vevs [64]. According to the discussion in section 5, the corrections due to complex Higgs vevs induced by the explicitly CP-violating phases can also be safely neglected in the MSSM [5].

In the NMSSM CP-violation enters the dispersion relations in several new ways. As already discussed in section 5, spontaneous CP-violation in the bubble wall occurs for specific values of the SUSY parameters, which leads to a variation of $\gamma, \delta$ and $\theta_{1,2}$. If this effect is present, it dominates the CP-violating part of the dispersion relations. Even in the absence of transitional CP-violation and with constant $\tan \beta$ there are contributions 

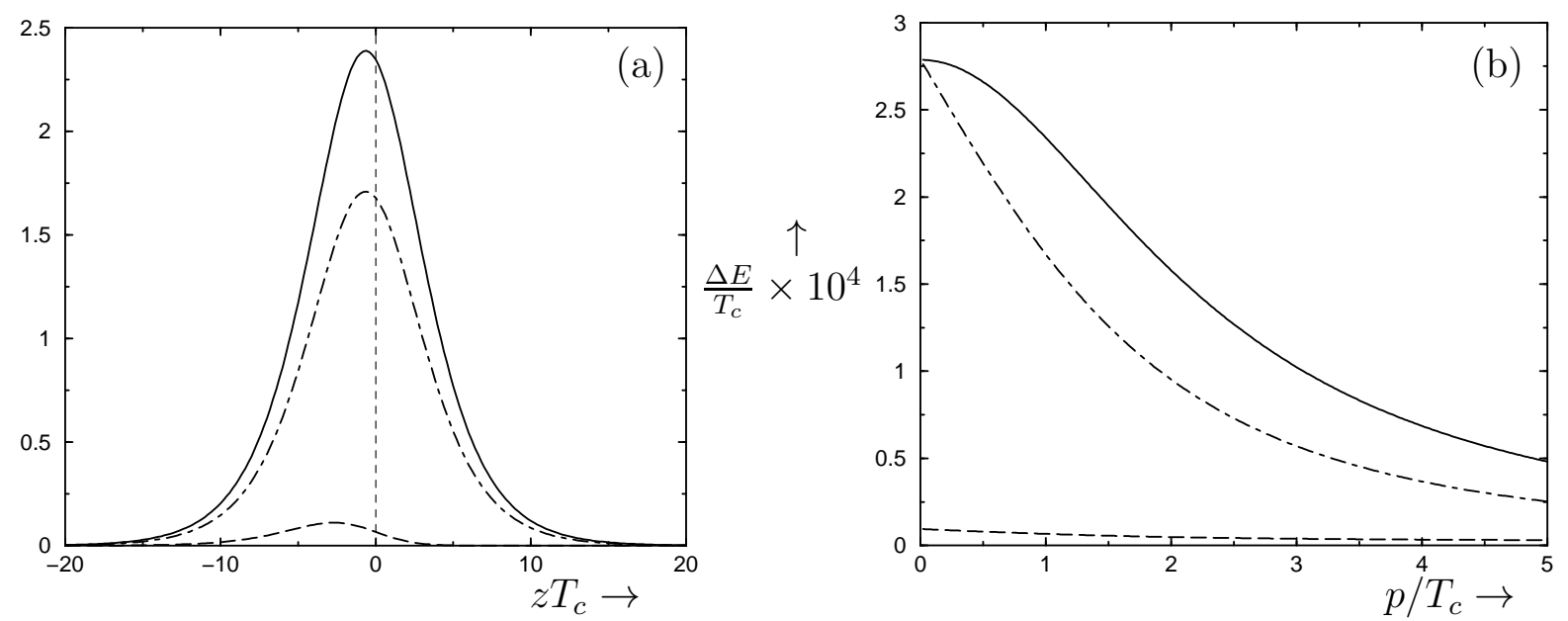

Figure 8: CP-violating contributions to the chargino dispersion relation for the explicitly CP-violating parameter set of fig. 6, (a) as a function of $z$ for $p=T_{c}$ and (b) as a function of $p$ for $z=0$. The dashed-dotted, dashed and solid lines represent the helicity and flavor contributions (6.40) and the result using the kinetic momentum (6.43), respectively.

to $\gamma^{\prime}$ and $\delta^{\prime}$ : The complex phases induced in the vevs by the explicitly CP-violating phases may considerably change in the bubble wall according to section 5 . Also the phase of the effective $\mu$-term, $\mu+\lambda S$, is position dependent because of the variation of the singlet vev. However, this effect is suppressed when the coupling $\lambda$ is small.

Again we calculate the (inverse) group velocity, now from (6.40). E-independent terms drop out, i.e. $\cosh ^{2} X_{I}$ can be substituted by $\sinh ^{2} X_{I}$. The kinetic momenta beyond the zeroth order $(6.29)$ then contain (order $\hbar)$ correction terms $\pm\left(\theta_{I}^{\prime}+\delta^{\prime} \sin ^{2} b-\right.$ $\left.\gamma^{\prime} \sin ^{2} a\right) m^{2} / 2 E\left(E^{2}-m^{2}\right)^{1 / 2}$. The dispersion relation can be inverted, and to leading order in the derivatives the CP-violating part of the dispersion relation for the eigenstate $L_{2}$ corresponding to $\tilde{h}_{2}^{+}$in the symmetric phase is

$$
\Delta E=-\operatorname{sign}\left(p_{z}\right)\left(\theta_{2}^{\prime}+\delta^{\prime} \sin ^{2} b-\gamma^{\prime} \sin ^{2} a\right) \frac{m_{2}^{2}}{2\left(p_{\text {kin }}^{2}+m_{2}^{2}\right)}
$$

where $m_{2}^{2}=\left|\left[M_{D}\right]_{22}\right|^{2}$ from eq. (6.42). For $p_{\text {kin }} \gg m$ this is $2\left|p_{z}\right| / p$ times the result one would obtain with canonical momentum after the substitution of $\cosh ^{2}$ by $\sinh ^{2}$ in (6.40) (see fig. 8 $\mathrm{b}$ ). Note that $\Delta E$ is now totally symmetric under the exchange of $H_{1}$ and $H_{2}$. This destroys the most prominent source term $\sim H_{1} H_{2}^{\prime}-H_{1}^{\prime} H_{2}$ of older work.

In fig. 8 we compare the $\mathrm{CP}$-violating contributions to the chargino dispersion relations stemming from the local helicity and flavor transformations (6.40) with the result from using kinetic variables (6.43). We assume that $p_{\perp}=0$, and approximate the bubble wall profile by a kink-ansatz with a common wall thickness for all fields, i.e. we take the straight connection between the symmetric and the broken minimum in field space. In this approximation $\tan \beta$ is automatically constant along the wall. We study the case of explicit CP-violation induced by $\phi_{\mu}=0.1$, which has already been considered in fig. 6 in section 5. It is characterized by $L_{w}=5 / T_{c}$ and $T_{c}=101 \mathrm{GeV}$. Fig. 8a shows the position dependence of $\Delta E$ for $p=p_{z}=T_{c}$. In fig. $8 \mathrm{~b}$ we present $\Delta E$ as a function of momentum for $z=0$. One observes that $\Delta E$ is dominated by the helicity contribution proportional to $\theta_{2}^{\prime}$ (dashed-dotted line), while the flavor contribution (dashed line) only gives a small 
correction. The result from the kinetic approach is larger by a factor between 1 and 2 . For the transitionally $\mathrm{CP}$-violating parameter set of fig. 7 the results are similar. The amplitude of $\Delta E$, however, is by a factor of about 7 larger, due to the larger amount of $\mathrm{CP}$-violation and the thinner bubble wall $\left(L_{w}=3 / T\right)$. In any case, $\Delta E$ provides only a small correction to dispersion relation.

We close this paragraph by briefly considering the case of Majorana fermions. Majorana spinors have the special property of being invariant under charge conjugation, i.e. particles and anti-particles are contained in the same four-component spinor. The mass matrix of Majorana particles is symmetric, and the entries are complex numbers, in general. In the NMSSM, the neutralinos are Majorana particles with a $5 \times 5$ mass matrix. From the symmetry property of the mass matrix we conclude $\mathbf{M} \mathbf{M}^{\dagger}=\mathbf{M} \mathbf{M}^{*}=\left(\mathbf{M}^{*} \mathbf{M}\right)^{*}$. The flavor transformations therefore obey $\mathbf{V}=\mathbf{U}^{*}$. Inserting this result into the general dispersions relations (6.36) (or into its two-dimensional version (6.40) ) we find that "left-handed" and "right-handed" states acquire exactly the opposite CP-violating contribution, in contrast to the general Dirac case. This is not surprising, since in case of Majorana fermions the two helicity states describe particles and anti-particles, respectively.

\subsubsection{The bosonic case}

In supersymmetric models the scalar superpartners of the top quarks may give important contributions to the CP-violating source that fuels baryon production. The stops efficiently interact with the bubble wall via the large top Yukawa coupling. They contain many degrees of freedom, and due to renormalization group flow the right-handed stop is probably lighter than any other squark. In the following we consider the interaction between the squarks and the bubble wall in the semi-classical limit. Our approximation is valid if the thickness of the bubble wall is much larger than the typical wavelength of particles in the plasma which is of order $1 / T$. As in the fermionic case, we derive dispersion relations in which the $\mathrm{CP}$-violating part is generated by varying phases in the scalar mass matrix.

Consider $N$ complex scalar fields $\mathbf{A}=\left(A_{1}, \ldots A_{N}\right)^{T}$ with mass term $\mathbf{A}^{\dagger} \mathbf{M}^{2} \mathbf{A}$. The entries of the hermitian mass matrix $\mathbf{M}^{2}$ may be complex. Due to the interaction with the wall, $\mathbf{M}^{2}$ depends on the position variable $z$. We represent $\mathbf{M}^{2}$ by a unitary matrix $\mathbf{U}$ and a real diagonal matrix $\mathbf{M}_{\mathbf{D}}^{2}=\operatorname{diag}\left(M_{D 1}^{2}, \ldots, M_{D N}^{2}\right)$

$$
\mathbf{M}^{2}=\mathbf{U M}_{\mathbf{D}}^{2} \mathbf{U}^{\dagger}
$$

Like in the fermionic case we omit the $N-1$ rotations in $\mathbf{U}$ which belong to the Abelian subgroup of $\mathrm{SU}(\mathrm{N})$. These transformations artificially redefine the interaction eigenstates. Their phases are not related to CP-violation, as can be deduced from the case of vanishing mixing. We then have a one to one correspondence between the $N^{2}$ real parameters in $\mathbf{M}^{2}$, and in $\mathbf{U}$ and $\mathbf{M}_{\mathbf{D}}^{2}$.

From the Klein-Gordon equation for the scalar fields $\left(\hbar^{2} \partial_{\mu} \partial^{\mu}+\mathbf{M}^{\mathbf{2}}\right) \mathbf{A}=0$ we obtain the equation of motion for the local mass eigenstates $\tilde{\mathbf{A}}=\mathbf{U}^{\dagger} \mathbf{A}$

$$
\left[-\hbar^{2}\left(\partial_{z}^{2}+2 \mathbf{U}^{\dagger} \partial_{z} \mathbf{U} \partial_{z}+\mathbf{U}^{\dagger} \partial_{z}^{2} \mathbf{U}\right)+\mathbf{M}_{\mathbf{D}}^{2}-E^{2}\right] \tilde{\mathbf{A}}(z)=0 .
$$

To derive this equation we implemented energy conservation in the wall frame by the ansatz $\tilde{\mathbf{A}}(t, z)=\tilde{\mathbf{A}}(z) e^{-i E t}$ and boosted to the Lorentz frame where there is no momentum parallel to the wall, i.e. $p_{\perp}=0$. 
We solve eq. (6.45) by the WKB method. To order $\mathcal{O}\left(\hbar^{0}\right)$ we can neglect the $\mathbf{U}^{\dagger} \partial_{z} \mathbf{U}$ and $\mathbf{U}^{\dagger} \partial_{z}^{2} \mathbf{U}$ contributions and find the wave functions $\tilde{\mathbf{A}}^{(I)}(z)=\mathbf{e}_{I} \exp \left(-i \int^{z} p_{z}\left(z^{\prime}\right) d z^{\prime}\right)$, where $\mathbf{e}_{I}$ is the $I$ th unit vector in flavor space. Furthermore, we obtain the dispersion relation $p_{z}^{2}=E^{2}-M_{D I}^{2}$. As we already found in the fermionic case, CP-violation vanishes in the classical limit.

We now include the order $\mathcal{O}\left(\hbar^{1}\right)$ corrections. We still can neglect the $\mathbf{U}^{\dagger} \partial_{z}^{2} \mathbf{U}$ term, while the $\mathbf{U}^{\dagger} \partial_{z} \mathbf{U}$ contribution becomes relevant now. Its off-diagonal entries reintroduce a coupling between the $N$ components of eq. (6.45). In the dispersion relation only the diagonal part of $\mathbf{U}^{\dagger} \partial_{z} \mathbf{U}$ enters. We obtain

$$
\tilde{\mathbf{A}}^{(I)}: E^{2}=M_{D I}^{2}+p_{z}^{2}+2 p_{z}\left[U^{\dagger} i \partial_{z} U\right]_{I},
$$

where $\left[U^{\dagger} i \partial_{z} U\right]_{I}$ denotes the $I$ th diagonal entry of the corresponding matrix. To transform the dispersion relations to a general Lorentz frame with non-vanishing $p_{\perp}$ one has to replace $E^{2}$ by $E^{2}-p_{\perp}^{2}$.

Let us evaluate the general expression (6.46) for the case $N=2$. Using the representation of $\mathbf{U}$ from eq. (6.38), we obtain

$$
\tilde{\mathbf{A}}^{(1,2)}: E^{2}=M_{D_{1,2}}^{2}+p_{z}^{2}-2 p_{z} \gamma^{\prime} \sin ^{2} a,
$$

Again, CP-violation arises due to the varying phase $\gamma$ in the transformation to the local mass eigenstates. A variation in a gives no contribution to the dispersion relations. For the anti-particles, $\tilde{\mathbf{A}}^{\dagger(1,2)}$, the CP-violating part in eq. (6.47) enters with the opposite sign. If we apply these expressions to the top squarks of the (N)MSSM with mass matrix

$$
\begin{aligned}
\mathcal{L} & =\cdots-\left(\tilde{t}^{*}, \tilde{t}^{c}\right)\left(\begin{array}{cc}
m_{L L}^{2} & m_{L R}^{2} \\
\left(m_{L R}^{2}\right)^{*} & m_{R R}^{2}
\end{array}\right)\left(\begin{array}{c}
\tilde{t} \\
\left(\tilde{t^{c}}\right)^{*}
\end{array}\right) \\
m_{L L}^{2} & =m_{Q_{3}}^{2}+y_{t}^{2}\left|H_{2}^{0}\right|^{2}+\left(\frac{g_{2}^{2}}{4}-\frac{g_{1}^{2}}{12}\right)\left(\left|H_{1}^{0}\right|^{2}-\left|H_{2}^{0}\right|^{2}\right) \\
m_{R R}^{2} & =m_{U_{3}}^{2}+y_{t}^{2}\left|H_{2}^{0}\right|^{2}+\frac{g_{1}^{2}}{3}\left(\left|H_{1}^{0}\right|^{2}-\left|H_{2}^{0}\right|^{2}\right) \\
m_{L R}^{2} & =y_{t}(\mu+\lambda S) H_{1}^{0}+y_{t} A_{t}^{*}\left(H_{2}^{0}\right)^{*},
\end{aligned}
$$

the parameters of $\mathbf{U}$ are given similarly to (6.41) by $\sin ^{2} a=2|A|^{2} / \Lambda(\Lambda+\Delta), \gamma=\arg A$ now with $A=y_{t}\left((\mu+\lambda S) H_{1}^{0}+A_{t}^{*} H_{2}^{0 *}\right)$. Solving (6.47) for $p$, one obtains $p(E)=\left(E^{2}-\right.$ $\left.M_{D_{1,2}}^{2}\right)^{1 / 2}+\gamma^{\prime} \sin ^{2} a$ to first order in $\gamma^{\prime}$. The group velocity $\left(\frac{\partial p}{\partial E}\right)^{-1}$ is independent of $\gamma^{\prime}$, thus stops do not contribute to $\mathrm{CP}$-violation in this order in the kinetic approach.

\subsection{Diffusion equations}

\subsubsection{The fluid approximation}

In this section we study the coupled differential equations that describe particle interactions and transport during the phase transition. We treat the plasma as consisting of quasi-classical particles with definite canonical position and momentum. This is an approximation to quantum Boltzmann equations which have to be discussed in principle. This picture is justified for thick walls $\left(p \gg 1 / L_{w}\right)$ if it predicts a sizable effect, not dominated by non-leading terms in the derivative expansion [65]. The dynamics of 
the particles is then governed by the dispersion relations $E(\vec{x}, \vec{p})$ derived in the previous section.

The information about the particle distributions is encoded in the phase space densities $f_{i}(\vec{x}, \vec{p}, t)$. Their temporal evolution follows from the Boltzmann equation

$$
d_{t} f_{i}=\left(\partial_{t}+\dot{\vec{x}} \cdot \partial_{\vec{x}}+\dot{\vec{p}} \cdot \partial_{\vec{p}}\right) f_{i}=\mathcal{C}_{i}[f]
$$

The time derivatives of position and momentum obey the Hamilton equations $\dot{\vec{x}}=$ $\partial_{\vec{p}} E(\vec{x}, \vec{p})$ and $\dot{\vec{p}}=-\partial_{\vec{x}} E(\vec{x}, \vec{p})$. The Boltzmann equation can in principle be solved numerically. However, to make it analytically tractable we use the fluid-type truncation 63

$$
f_{i}(\vec{x}, \vec{p}, t)=\frac{1}{e^{\beta\left(E_{i}-v_{i} p_{z}-\mu_{i}\right)} \pm 1}
$$

for the phase space densities of fermions $(+)$ and bosons $(-)$ in the rest frame of the plasma. Here $v_{i}$ and $\mu_{i}$ denote the velocity perturbations and chemical potentials for each fluid, respectively. We also split $E_{i}$ into a dominant part $E_{0 i}=\sqrt{p^{2}+m_{i}^{2}}$ and a perturbation $\Delta E_{i} \sim \partial_{z} \theta$ which is related to $\mathrm{CP}$-violation. The chemical potentials are the central quantities that finally will determine the baryon asymmetry. The velocity perturbation on the other hand, is only introduced to allow the particles to move in response to the force, giving rise to chemical potential perturbations. The fluid truncation is valid as long as perturbations beyond the ansatz (6.50) are attenuated faster than chemical potential perturbations. As discussed in ref. [63] this requires $v_{w}<\frac{L_{w}}{3 D}$, where the diffusion constant $D$ will be introduced below.

We are looking for a "stationary" solution of the Boltzmann equation, because at late times the wall moves with constant velocity $v_{w}$. This means that any explicit time dependence enters in the combination $\bar{z} \equiv z-v_{w} t$. Inserting the fluid ansatz into the Boltzmann equation (6.49) we obtain to linear order in the perturbations $\Delta E_{i}, \mu_{i}$ and $v_{i}$

$$
f_{ \pm}^{\prime}\left\{-v_{w}\left(\Delta E_{i}^{\prime}-v_{i}^{\prime} p_{z}-\mu_{i}^{\prime}\right)-\frac{p_{z}}{E_{0 i}}\left(v_{i}^{\prime} p_{z}+\mu_{i}^{\prime}\right)+\frac{\left(m_{i}^{2}\right)^{\prime}}{2 E_{0 i}} v_{i}\right\}=\mathcal{C}_{i}[f]
$$

where $f_{ \pm}^{\prime}=d f_{ \pm} / d E_{0}=-\beta e^{\beta E_{0}} /\left(e^{\beta E_{0}} \pm 1\right)^{2}$ denotes the derivative of the unperturbed Fermi-Dirac or Bose-Einstein distribution. The remaining primes in eq. (6.51) denote $\partial_{\bar{z}}$. More precisely, eq. 6.51) is the difference of the corresponding equations for particles and anti-particles. The parameters $\Delta E_{i}, \mu_{i}$ and $v_{i}$ therefore represent the differences of these quantities for particles and anti-particles. For that reason the term $f_{ \pm}^{\prime} v_{w} E_{0 i}^{\prime}$, which provides the main contribution to friction in the calculation of the wall velocity [67, cancels out. In order to obtain differential equations for the perturbations we average over momentum, weighting eq. (6.51) by 1 and $p_{z}$, respectively, and receive

$$
\begin{aligned}
-\left\langle\frac{p_{z}^{2}}{E_{0 i}}\right\rangle v_{i}^{\prime}+\kappa_{i} v_{w} \mu_{i}^{\prime} & =\left\langle\mathcal{C}_{i}\right\rangle, \\
v_{w}\left\langle p_{z}^{2}\right\rangle v_{i}^{\prime}-\left\langle\frac{p_{z}^{2}}{E_{0 i}}\right\rangle \mu_{i}^{\prime}-v_{w}\left\langle p_{z} \Delta E_{0 i}^{\prime}\right\rangle & =\left\langle p_{z} \mathcal{C}_{i}\right\rangle .
\end{aligned}
$$

The average is defined according to

$$
\langle\cdot\rangle \equiv \frac{\int d^{3} p f_{ \pm}^{\prime}(\cdot)}{\int d^{3} p f_{+}^{\prime}(m=0)} \equiv \kappa_{i} \frac{\int d^{3} p f_{ \pm}^{\prime}(\cdot)}{\int d^{3} p f_{ \pm}^{\prime}} .
$$


The statistical factor $\kappa$ is 1 for massless fermions, 2 for massless bosons and exponentially small for particles much heavier than $T$. In the derivation of eqs. (6.52) and (6.53) one uses that the energy perturbations $\Delta E_{i}$ are odd in $p_{z}$, according to the results of the previous section. Furthermore, we neglected the contribution of the last term in the curly brackets of eq. (6.51) which turns out to be numerically less important than the $\left\langle\frac{p_{z}^{2}}{E_{0 i}}\right\rangle v_{i}^{\prime}$ term taken into account in eq. (6.52).

To linear order in the perturbations the collision terms on the RHS of the Boltzmann equation take the form 64

$$
\left\langle\mathcal{C}_{i}\right\rangle=\sum_{p} \Gamma_{p}^{d} \sum_{j} \mu_{j}, \quad\left\langle p_{z} \mathcal{C}_{i}\right\rangle=v_{i} \bar{p}_{z}^{2} \sum_{p} \Gamma_{p}^{e}
$$

where $\Gamma_{p}$ denotes the rate of the process $p$. In the sum over chemical potentials, incoming particles enter with positive sign, outgoing particles with negative sign. We distinguish between elastic interactions with rates $\Gamma^{e}$ and decays (inelastic interactions) with rates $\Gamma^{d}$. Since elastic scattering conserves the number of particles these processes do not contribute to $\left\langle\mathcal{C}_{i}\right\rangle$. In the hot electroweak plasma the elastic processes dominated by gauge boson exchange reactions are much more efficient than the particle decays coming from Yukawa interactions, sphalerons, etc. It is therefore justified to neglect the contribution of inelastic processes to $\left\langle p_{z} \mathcal{C}_{i}\right\rangle$.

In the collision integral a delta function $\delta\left(\sum p_{i}\right)$ usually represents the conservation of energy and momentum in the interactions. However, in the plasma frame this is no longer true, because of the moving bubble wall. The Boltzmann equation is formulated in terms of mass eigenstates which change in space and time due to the varying Higgs (and singlet) vevs. As a result, additional CP-violating contributions to the collision term arise, which are related to what has been dubbed "spontaneous" baryogenesis [70]. The final form of the collision term is therefore given by 64

$$
\left\langle\mathcal{C}_{i}\right\rangle=\sum_{p} \Gamma_{p}^{d}\left(\sum_{j} \mu_{j}+\Delta E_{\mathrm{sp}, p}\right)
$$

where $\Delta E_{\mathrm{sp}, p}$ denotes the bubble wall induced deviation from energy conservation in the corresponding process.

Finally, we reduce the two coupled transport equations (6.52) and (6.53) to a single one by differentiating (6.53) and eliminating $v_{i}$ in favor of $\mu_{i}$. We approximate the thermal averages according to $\left\langle p_{z}^{2} / E_{0}\right\rangle \sim \kappa_{i}\left\langle p_{z}^{2} / E_{0}\right\rangle_{0},\left\langle p_{z}^{2}\right\rangle \sim \kappa_{i}\left\langle p_{z}^{2}\right\rangle_{0}$, where the subscript "0" denotes averaging with the massless, unperturbed Fermi-Dirac distribution. Defining the diffusion constant $D_{i}=\kappa_{i}\left\langle p_{z}^{2} / E_{0}\right\rangle_{0}^{2} /\left(\bar{p}_{z}^{2} \Gamma_{i}^{e}\right)$ we find the diffusion equation [64]

$$
\begin{gathered}
-\kappa_{i}\left(D_{i} \mu_{i}^{\prime \prime}+v_{w} \mu_{i}^{\prime}\right)+\sum_{p} \Gamma_{p}^{d} \sum_{j} \mu_{j}=S_{i}, \\
S_{i}=\frac{D_{i} v_{w}}{\left\langle p_{z}^{2} / E_{0}\right\rangle_{0}}\left\langle p_{z} \Delta E_{i}^{\prime}\right\rangle^{\prime}-\sum_{p} \Gamma_{p}^{d}\left\langle\Delta E_{\mathrm{sp}, p}\right\rangle .
\end{gathered}
$$

In order to obtain eqs. (6.57) we neglected derivatives of the CP-conserving thermal averages and rates, and left aside ratios of inelastic to elastic scatterings. We also ignored terms beyond leading order in the wall velocity, an approximation that certainly breaks 
down if the wall velocity approaches the speed of sound in the plasma, $v_{s}=1 / \sqrt{3} \sim 0.58$. If the wall moves faster than $v_{s}$, perturbations cannot propagate in the region in front of the wall any more. Notice that the first contribution to the CP-violating source term $S_{i}$ in (6.57), which is due to the semi-classical force, is proportional to the diffusion constant. This is simply because particles must move in order to build up perturbations. The second, "spontaneous" source term is independent of transport properties of the corresponding particles and therefore dominates in the limit of inefficient transport. In $S_{i}$ the thermal averages over the $\mathrm{CP}$-violating energy perturbations $\Delta E$ are performed using the massive distribution functions in order to account for Boltzmann suppression of heavy particles.

\subsubsection{Diffusion equations for supersymmetric models}

In the previous paragraph we derived a complicated network of diffusion equations (6.57) that couples all particle species in the hot plasma. In principle, after specification of decay rates, diffusion constants and $\mathrm{CP}$-violating sources, it is possible to solve the transport equations numerically. However, analytic progress can be made by using conservation laws and neglecting interactions that are slow compared to the relevant time scale. An interaction with rate $\Gamma$ can be neglected if the typical interaction time is large compared to the average time a particle spends diffusing in front of the wall before being caught by the wall, which is equivalent to 63.

$$
\frac{D}{v_{w}^{2}} \ll \Gamma^{-1}
$$

The electroweak ("weak") sphaleron interaction with rate $\Gamma_{w s}$ is slow in precisely the sense of (6.58) (unless the wall velocity is particularly small, i.e. $v_{w}<\sim 0.01$ ). In the following we will therefore assume baryon and lepton number conservation and include the weak sphalerons only at the end of the calculation. The neglect of the weak sphalerons allows us to completely forget about leptons in our transport equations and compute only the quark and Higgs densities.

The processes we do take into account are the supergauge interactions, the strong sphaleron interactions, and those described by the Lagrangian

$$
\mathcal{L}_{\text {int }}=y_{t} t^{c} q_{3} H_{2}+y_{t} \tilde{t}^{c} q_{3} \tilde{h}_{2}+y_{t} t^{c} \tilde{q}_{3} \tilde{h}_{2}-y_{t} \mu \tilde{t}^{c *} \tilde{q}_{3}^{*} H_{1}+y_{t} A_{t} \tilde{t}^{c} \tilde{q}_{3} H_{2}+\text { h.c. }
$$

Via terms of type $\lambda \tilde{s} \tilde{h}_{1} H_{2}$, the singlino, $\tilde{s}$, is coupled to the quark-Higgs system. In the case of transitional CP-violation triggered in the singlet sector (see fig. 7), the singlino receives the largest $\mathrm{CP}$-violating source term of all particles, even though its impact on baryogenesis is suppressed by the small coupling $\lambda$. For the sake of simplicity we ignore this interesting contribution in the following. We assume the supergauge interactions to be in equilibrium. The chemical potential of any particle is then equal to that of its superpartner, with exception of the singlet field. It is convenient to define the chemical potentials $\mu_{U}=\left(\mu_{u^{c}}+\mu_{\tilde{u}^{c}}\right) / 2, \mu_{Q_{1}}=\left(\mu_{u}+\mu_{d}+\mu_{\tilde{u}}+\mu_{\tilde{d}}\right) / 4, \mu_{H_{1}}=\left(\mu_{H_{1}^{0}}+\mu_{H_{1}^{-}}+\mu_{\tilde{h}_{1}^{0}}+\mu_{\tilde{h}_{1}^{-}}\right) / 4$, etc. In this notation the interaction terms take the form

$$
\begin{gathered}
\left(\Gamma_{y}+\Gamma_{y A}\right)\left(\mu_{H_{2}}+\mu_{Q_{3}}+\mu_{T}\right), \quad \Gamma_{y \mu}\left(\mu_{H_{1}}-\mu_{Q_{3}}-\mu_{T}\right), \\
\Gamma_{s s}\left(2 \mu_{Q_{3}}+2 \mu_{Q_{2}}+2 \mu_{Q_{1}}+\mu_{T}+\mu_{B}+\mu_{C}+\mu_{S}+\mu_{U}+\mu_{D}\right), \\
\Gamma_{h f}\left(\mu_{H_{1}}+\mu_{H_{2}}\right), \quad \Gamma_{m}\left(\mu_{Q_{3}}+\mu_{T}\right), \quad \Gamma_{H_{1}} \mu_{H_{1}}, \quad \Gamma_{H_{2}} \mu_{H_{2}} .
\end{gathered}
$$

\footnotetext{
${ }^{13}$ In contrast to refs. [58, 64] we count all left-handed particles and the corresponding superpartners with positive signs.
} 
The rates in the first line are related to the interactions $(6.59) . \Gamma_{s s}$ denotes the strong sphaleron rate. $\Gamma_{h f}$ is due to Higgsino helicity flips induced by the $\mu \tilde{h}_{1} \tilde{h}_{2}$ term. $\Gamma_{H_{1,2}}$ and $\Gamma_{m}$ correspond to Higgs and axial top number violating processes, present only in the phase boundary and the broken phase.

Because of the small Yukawa couplings of the first and second family quarks, these particles are in very good approximation only produced by strong sphalerons. Hence their number densities are algebraically constrained. If the system is near thermal equilibrium, number densities and chemical potentials are related by

$$
n_{i}=\frac{1}{6} k_{i} \mu_{i} T^{2}
$$

where $k_{i}$ is the appropriate sum over statistical factors $\kappa$ introduced in (6.54), e.g. $k_{Q_{1}}=$ $N_{c}\left(\kappa_{u}+\kappa_{d}+\kappa_{\tilde{u}}+\kappa_{\tilde{d}}\right), k_{U}=N_{c}\left(\kappa_{u^{c}}+\kappa_{\tilde{u}^{c}}\right), k_{H_{1}}=\left(\kappa_{H_{1}^{0}}+\kappa_{H_{1}^{-}}+\kappa_{\tilde{h}_{1}^{0}}+\kappa_{\tilde{h}_{1}^{-}}\right)$, etc. $N_{c}=3$ denotes the number of colors. In the massless limit used in ref. [64 one obtains $k_{Q_{1,2,3}}=18$, $k_{U}=k_{D}=\ldots=k_{T}=9, k_{H_{1,2}}=6$. Using baryon number conservation the strong sphaleron rate reads

$$
\Gamma_{s s}\left(2 \mu_{Q_{3}}+\cdots+\mu_{D}\right)=\Gamma_{s s}\left[\left(2+9 \frac{k_{Q_{3}}}{k_{B}}\right) \mu_{Q_{3}}+\left(1-9 \frac{k_{T}}{k_{B}}\right) \mu_{T}\right]
$$

To arrive at this expression we assumed that all the squark partners of the light quarks are degenerate in mass. Assuming equilibrium for the strong sphalerons we obtain

$$
\mu_{T}=\frac{2 k_{B}+9 k_{Q_{3}}}{9 k_{T}-k_{B}} \mu_{Q_{3}} .
$$

The validity of this assumption will be discussed below.

We are now able to write down the reduced set of diffusion equations for the relevant particle species $Q_{3}, H_{1}$ and $H_{2}$

$$
\begin{aligned}
& -A \mathcal{D}_{q} \mu_{Q_{3}}+\left(\Gamma_{y}+\Gamma_{y A}\right)\left[\mu_{H_{2}}+B \mu_{Q_{3}}\right]-\Gamma_{y \mu}\left[\mu_{H_{1}}-B \mu_{Q_{3}}\right]+B \Gamma_{m} \mu_{Q_{3}}=S_{Q_{3}} \\
& -k_{H_{1}} \mathcal{D}_{h} \mu_{H_{1}}+\Gamma_{y \mu}\left[\mu_{H_{1}}-B \mu_{Q_{3}}\right]+\Gamma_{h f}\left[\mu_{H_{1}}+\mu_{H_{2}}\right]+\Gamma_{H_{1}} \mu_{H_{1}}=S_{H_{1}} \\
& -k_{H_{2}} \mathcal{D}_{h} \mu_{H_{2}}+\left(\Gamma_{y}+\Gamma_{y A}\right)\left[\mu_{H_{2}}+B \mu_{Q_{3}}\right]+\Gamma_{h f}\left[\mu_{H_{1}}+\mu_{H_{2}}\right]+\Gamma_{H_{2}} \mu_{H_{2}}=S_{H_{2}}
\end{aligned}
$$

where

$$
\begin{aligned}
A & =\frac{9 k_{T} k_{Q_{3}}+9 k_{B} k_{Q_{3}}+4 k_{B} k_{T}}{9 k_{T}-k_{B}} \\
B & =\frac{k_{B}+9 k_{T}+9 k_{Q_{3}}}{9 k_{T}-k_{B}}
\end{aligned}
$$

and $\mathcal{D}_{i} \equiv D_{i} \frac{d^{2}}{d \bar{z}^{2}}+v_{w} \frac{d}{d \bar{z}}$. These equations result from summing the diffusion equations of particles which belong to the same color and $\mathrm{SU}(2)$ multiplets. We have taken a common diffusion constant, $D_{q}$, for the (s)quarks, as well as one for the two Higgs doublets, $D_{h}$. Notice that the effects of hypercharge screening have been neglected, which can be shown to affect the created baryon asymmetry at most by a factor of order one [72].

We keep the rates related to the top Yukawa interactions finite. If these interaction are in equilibrium, the resulting diffusion equations are sourced only by the combination 
$S_{H_{1}}-S_{H_{2}}$, because of the constraint $\mu_{H_{1}}+\mu_{H_{2}}=0$. As a result the dominant contribution to the chargino source terms cancels, because the corresponding terms for $\tilde{h}_{1}^{-}$and $\tilde{h}_{2}^{+}$are exactly of the same size. This would not be true for the $\gamma^{\prime}, \delta^{\prime}$ contribution if we used the dispersion relations for canonical momenta.

In the MSSM the full diffusion equations (6.64) have already been studied in ref. [71] and later in ref. [73]. Applying the closed time path formalism to calculate the source terms, the diffusion equations (6.64) were analyzed recently in ref. 74].

\subsection{The baryon asymmetry}

\subsubsection{Solution of the diffusion equations}

In the previous section we derived differential equations for the chemical potentials of the various particle species contained in the hot electroweak plasma. Baryon number violation has been neglected throughout this calculation. However, what we set out to compute was the total baryon asymmetry created during the phase transition. So before solving the network of diffusion equations, we turn to baryon number generation by weak sphaleron processes, which is fueled by the chemical potential of left-handed quarks, $\mu_{\mathcal{B}_{L}} \equiv \mu_{Q_{1}}+\mu_{Q_{2}}+\mu_{Q_{3}}$. Using baryon number conservation and eq. (6.63) we find

$$
\mu_{\mathcal{B}_{L}}=\left[1-\frac{k_{Q_{3}}+2 k_{T}}{9 k_{T}-k_{B}}\left(\frac{2 k_{B}}{k_{Q_{1}}}+\frac{2 k_{B}}{k_{Q_{2}}}\right)\right] \mu_{Q_{3}} \equiv C \mu_{Q_{3}} .
$$

The evolution of the baryon number density $n_{\mathcal{B}}$ is governed by

$$
-\mathcal{D}_{q} n_{\mathcal{B}}+3 \Theta(\bar{z}) \Gamma_{w s}\left(T^{2} \mu_{\mathcal{B}_{L}}-a n_{\mathcal{B}}\right)=0
$$

where we have assumed identical diffusion constants for all quarks and squarks, and neglected contributions of leptons. The position dependence of the weak sphaleron rate is modeled by a step function $\Theta(\bar{z})$ : anomalous baryon number violation is unsuppressed in the symmetric phase $(\bar{z}>0)$ and suddenly switched off in the broken phase $(\bar{z}<0)$. The second term in Eq. (6.67) describes damping of the baryon asymmetry by weak sphalerons in the symmetric phase. The parameter $a$ depends on the degrees of freedom present in the hot plasma. Taking only the right-handed stop to be light gives $a=48 / 7$ [71].

From Eq. 6.67 one can easily obtain the baryon to entropy ratio in the broken phase

$$
\eta_{B} \equiv \frac{n_{\mathcal{B}}}{s}=\frac{135 \Gamma_{w s}}{2 \pi^{2} g_{*} v_{w} T} \int_{0}^{\infty} d \bar{z} \mu_{\mathcal{B}_{L}}(\bar{z}) e^{-\nu \bar{z}}
$$

where we have taken the entropy density $s=\left(2 \pi^{2} g_{*} / 45\right) T^{3}$ and $\nu=3 a \Gamma_{w s} /\left(2 v_{w}\right)$ [71. $g_{*} \sim 126$ is the effective number of degrees of freedom at the phase transition temperature. Eq. (6.68) shows that the integral over the left-handed quark number, $n_{L} \propto \mu_{\mathcal{B}_{L}}$ in the symmetric phase determines the final baryon asymmetry.

We now return to eqs. (6.64) in order to compute $\mu_{\mathcal{B}_{L}}$. These linear second order differential equations can be solved by finding the appropriate Green's function. We keep the discussion general and consider the following set of $N$ coupled diffusion equations

$$
\left(\begin{array}{ccc}
-k_{11} \mathcal{D}_{11}+\Gamma_{11} & \cdots & -k_{1 N} \mathcal{D}_{1 N}+\Gamma_{1 N} \\
\vdots & \ddots & \vdots \\
-k_{N 1} \mathcal{D}_{N 1}+\Gamma_{N 1} & \cdots & -k_{N N} \mathcal{D}_{N N}+\Gamma_{N N}
\end{array}\right)\left(\begin{array}{c}
\mu_{1} \\
\vdots \\
\mu_{N}
\end{array}\right)=\left(\begin{array}{c}
S_{1} \\
\vdots \\
S_{N}
\end{array}\right)
$$


where $\mathcal{D}_{a b}=D_{a b} \frac{d^{2}}{d \bar{z}^{2}}+v_{w} \frac{d}{d \bar{z}}$. The corresponding boundary conditions read $\mu_{a}(|\bar{z}| \rightarrow \infty)=$ 0 . The matrix valued Green's function $G_{a b}$ is defined by $\sum_{c=1}^{N}\left(-k_{a c} \mathcal{D}_{a c}+\Gamma_{a c}\right) G_{c b}(\bar{z})=$ $\delta_{a b} \delta(\bar{z})$. In the transport equations (6.64) also position dependent rates are present, e.g. $\Gamma_{m}$. They typically vanish in the symmetric phase and become maximal in the broken phase. In order to keep the problem analytically tractable we simply model the position dependence of these rates by step functions, i.e. $\Gamma_{a b}(\bar{z})=\Gamma_{+a b} \Theta(\bar{z})+\Gamma_{-a b} \Theta(-\bar{z})$.

The general structure of the Green's function then reads

$$
G_{a b}(\bar{z})=\Theta(\bar{z}) \sum_{i=1}^{N} c_{i+a b} e^{-\lambda_{i+} \bar{z}}+\Theta(-\bar{z}) \sum_{i=1}^{N} c_{i-a b} e^{-\lambda_{i-} \bar{z}} .
$$

The constants $\lambda_{i \pm}$ can be computed from $\operatorname{det}\left[k_{a b}\left(-D_{a b} \lambda_{ \pm}^{2}+v_{w} \lambda_{ \pm}\right)+\Gamma_{ \pm a b}\right]=0$. Of course, this procedure supplies us with $4 N$ solutions. However, $2 N$ of them $\left(\lambda_{i+}<0\right.$ for $\bar{z}>0$ and $\lambda_{i-}>0$ for $\bar{z}>0$ ) correspond to exponentially growing solutions of eq. (6.69) and have to be discarded. The coefficients $c_{i \pm a b}$ can then be computed from the $2 N^{3}$ dimensional set of linear equations

$$
\begin{aligned}
\sum_{b=1}^{N}\left[-k_{a b} D_{a b} \lambda_{i+}^{2}+k_{a b} v_{w} \lambda_{i+}+\Gamma_{+a b}\right] c_{i+b c} & =0 \\
\sum_{b=1}^{N}\left[-k_{a b} D_{a b} \lambda_{i-}^{2}+k_{a b} v_{w} \lambda_{i-}+\Gamma_{-a b}\right] c_{i-b c} & =0 \\
\sum_{i=1}^{N}\left[c_{i+a b}-c_{i-a b}\right] & =0 \\
\sum_{i, b=1}^{N} k_{a b} D_{a b}\left[\lambda_{i+} c_{i+b c}-\lambda_{i-} c_{i-b c}\right] & =\delta_{a c}
\end{aligned}
$$

where $\Gamma_{ \pm a b}$ denote the rates for positive and negative $\bar{z}$. Eqs. (6.71) and (6.72) result from solving the homogeneous version of eq. (6.69) in the range of positive and negative $\bar{z}$, respectively. Only $2 N^{2}(N-1)$ of them are independent. The continuity of the Green's function at $\bar{z}=0$ is guaranteed by eq. (6.73). Finally, eq. (6.74) is obtained by integrating the definition equation for the Green's function on an infinitesimally small interval around $\bar{z}=0$. Diffusion constants $D_{a b}$ and statistical factors $k_{a b}$ differing in broken and symmetric phase can be treated along the same lines.

Once the Green's function is known one can easily compute the chemical potentials. Applying the general formulas to the diffusion equations (6.64) we identify $\mu_{1} \equiv \mu_{Q_{3}}$, $\mu_{2} \equiv \mu_{H_{1}}, \mu_{3} \equiv \mu_{H_{2}}, S_{1} \equiv S_{Q_{3}}, S_{2} \equiv S_{H_{1}}$ and $S_{3} \equiv S_{H_{2}}$. Using eqs. (6.68) and (6.66) we obtain for the baryon to entropy ratio

$$
\eta_{B}=\frac{135 \Gamma_{w s}}{2 \pi^{2} g_{*} v_{w} T} C \int_{0}^{\infty} d \bar{z} e^{-\nu \bar{z}} \int_{-\infty}^{\infty} d \bar{z}^{\prime} G_{1 a}\left(\bar{z}-\bar{z}^{\prime}\right) S_{a}\left(\bar{z}^{\prime}\right) .
$$

Since the Green's function consists only of exponentials, the $\bar{z}$-integration can be performed analytically. The evaluation of this expressions is performed in the next paragraph. Also the various approximations we used in its derivation will be discussed. 


\subsubsection{Numerical evaluation and discussion}

Before starting to calculate a numerical value for the emerging baryon asymmetry we discuss the validity of the assumptions and approximations made in the derivation of eq. (6.75). This requires the specification of the various parameters that enter the diffusion equations. For the diffusion constants of quark and Higgs fields we take [74]

$$
D_{q}=\frac{6}{T}, \quad D_{h}=\frac{110}{T}
$$

We use the rates [74]

$$
\begin{array}{r}
\Gamma_{y}+\Gamma_{y A}=0.015 T, \quad \Gamma_{y \mu}=0, \quad \Gamma_{m}=0.05 T \Theta(-\bar{z}) \\
\Gamma_{h f}=0.016 T, \quad \Gamma_{H_{1}}=\Gamma_{H_{2}}=0.0036 T \Theta(-\bar{z}) .
\end{array}
$$

$\Gamma_{y \mu}$ is strongly suppressed because it involves heavy left-handed stop states (6.59). $\Gamma_{m}$, $\Gamma_{H_{1}}$ and $\Gamma_{H_{2}}$ are present only broken phase. We model their position dependence by a step function. The weak and strong sphaleron rates are given by 76

$$
\begin{aligned}
\Gamma_{w s} & \approx 6 \alpha_{w}^{4} T \approx 2.2 \times 10^{-5} T \\
\Gamma_{s s} & \approx 1500 \Gamma_{w s} \approx 0.033 T .
\end{aligned}
$$

It has been shown recently that parametrically $\Gamma_{w s}=C \ln \left(1 / g^{2}\right) \alpha_{w}^{5}$ [77], but lattice measurements of the rate are consistent with $C \sim 1 / \alpha_{w}$. The thickness of the bubble wall varies considerably in the NMSSM, $1 / T \lesssim L_{w} \lesssim 20 / T$, i.e. the wall may become much thinner than in case of the MSSM, where $20 / T \lesssim L_{w} \lesssim 30 / T$ has been found [35]. Calculations of the wall velocity in the SM lead to $0.36<v_{w}<0.44$ 67. Gauge fields in the hot plasma diminish this result [66]. In supersymmetric models there arise additional friction terms from the SUSY particles, in the first place from a light top squark. In the MSSM, this can bring down the wall velocity to $v_{w} \sim 10^{-2}$ 68. In the following we treat $v_{w}$ as a free parameter and examine its impact on the emerging baryon asymmetry.

Let us now summarize the approximations leading to eq. (6.75) for the baryon to entropy ratio:

- Assumption 1: $L_{w}>\frac{1}{T}$ so that most particles in the plasma are indeed accurately described by the WKB approximation in their interaction with the bubble wall. For typical wall thicknesses in the NMSSM, $5 / T \lesssim L_{w} \lesssim 15 / T$, this assumption is very well justified, although for the very thinnest walls $L_{w} \sim 1 / T$ the WKB approximation becomes questionable.

- Assumption 2: $v_{w}<\frac{L_{w}}{3 D}$, the thermalization condition that guarantees the applicability of the fluid ansatz. In case of (s)quarks this condition is satisfied for $v<0.4$ even for rather thin walls with $L_{w} \sim 5 / T$. Higgs particles thermalize much slower, as can be deduced from their large diffusion constant. Even for the largest wall thicknesses, $L_{w} \sim 20 / T$, rather small velocities, $v_{w}<0.1$, are required.

- Assumption 3: $v_{w}<\frac{1}{\sqrt{3}}$. We work to linear order in the wall velocity, which is only justified if the wall moves slower than the speed of sound in the plasma. Otherwise, diffusion in the region in front of the wall, giving rise to "non-local" baryogenesis, is no longer possible. However, in case of $v_{w}>\frac{1}{\sqrt{3}}$ the fluid approximation would break down anyway, i.e. from assumption 3 no new constraints result. 
- Assumption 4: $\Gamma_{w s}<v_{w}^{2} / D$, so that the back-reaction of the baryon number violating processes can be neglected in the diffusion equations (6.64). According to eqs. (6.76) and (6.78) this requires $v_{w}>0.01$ (quarks) and $v_{w}>0.04$ (Higgs particles).

- Assumption 5: $\Gamma_{s s}>v_{w}^{2} / D$ in order to put the strong sphaleron interaction to equilibrium. In this case the strongest constraint, $v_{w}<0.45$, result from the quarks, which is easily satisfied.

The calculation of the final baryon asymmetry still requires the specification of the source terms (6.57) which enter eq. (6.75). We concentrate on the source terms induced by the semi-classical force and neglect those related to spontaneous baryogenesis. We include the source terms of the top quark (6.32) and the charged Higgsinos (6.40). For sake of simplicity we neglected the neutral Higgsinos, which should affect the final baryon asymmetry at most by a factor of order one. Also the singlino is disregarded.

In order to evaluate the source terms $(6.57)$ the bubble wall profile is required. We approximate our numerical solutions by a kink-ansatz with a common wall thickness for all fields present in the bubble. However, we allow for a variation in $\tan \beta$ in the bubble wall by taking $\left|H_{2}^{0}(z)\right|=\left|H_{1}^{0}(z)\right|\left(\tan \beta_{0}+\right.$ const $\left.\cdot\left|H_{1}^{0}(z)\right|^{2} \mid\right)$, in the spirit of fig. 5a. The relevant source terms are obtained from combining eqs. (6.32), (6.40) and (6.57)

$$
\begin{aligned}
S_{t}= & \frac{N_{c} D_{q} v_{w}}{\left\langle p_{z}^{2} / E\right\rangle_{0}}\left(\langle A\rangle \theta_{t}^{\prime \prime}+\langle B\rangle\left(m_{t}^{2}\right)^{\prime} \theta_{t}^{\prime}\right)^{\prime} \\
S_{\tilde{h}_{1}}= & \frac{D_{h} v_{w}}{\left\langle p_{z}^{2} / E\right\rangle_{0}}\left(\langle A\rangle\left(\theta_{\tilde{h}}^{\prime}-\gamma_{\tilde{h}}^{\prime} \sin ^{2}\left(a_{\tilde{h}}\right)+\delta_{\tilde{h}}^{\prime} \sin ^{2}\left(b_{\tilde{h}}\right)\right)^{\prime}+\right. \\
& \left.\quad+\langle B\rangle\left(m_{\tilde{h}}^{2}\right)^{\prime}\left(\theta_{\tilde{h}}^{\prime}-\gamma_{\tilde{h}}^{\prime} \sin ^{2}\left(a_{\tilde{h}}\right)+\delta_{\tilde{h}}^{\prime} \sin ^{2}\left(b_{\tilde{h}}\right)\right)\right) ! \\
S_{\tilde{h}_{2}}= & S_{\tilde{h}_{1}}
\end{aligned}
$$

with

$$
\langle A\rangle=\left\langle\frac{\left|p_{z}\right||m|^{2}}{2 E^{2}}\right\rangle_{+}\langle B\rangle=\left\langle\frac{\left|p_{z}\right|\left(E^{2}-|m|^{2}\right)}{2 E^{4}}\right\rangle_{+}
$$

Here $N_{c}=3$ is the number of colors and $E^{2}=\vec{p}^{2}+m_{i}^{2}, E_{z}^{2}=p_{z}^{2}+m_{i}^{2}$ and $p_{\perp}^{2}=\vec{p}^{2}-p_{z}^{2}$. With exception of $\left\langle p_{z}^{2} / E\right\rangle_{0}$ all thermal averages in eqs. (6.79) - (6.81) are performed with massive distribution functions for fermions (6.54), which ensures the decoupling of heavy particles. Taking into account only the source terms of eqs. (6.79) - (6.80), we have $S_{Q_{3}}=S_{t}, S_{H_{1}}=S_{\tilde{h}_{1}}$ and $S_{H_{2}}=S_{\tilde{h}_{2}}$.

In the approach with canonical momenta (which we had in the first version of this paper), there was a source $S_{\tilde{t}}$ for the stop and $S_{\tilde{h}_{1}} \neq S_{\tilde{h}_{2}}$. The latter leads to an important contribution to the $H_{1}-H_{2}$-combination, the well-known term proportional $(\tan \beta)^{\prime}$ and to an additional term for the singlet field. These are absent now. The brackets $\langle A\rangle$ and $\langle B\rangle$ in the approach with canonical momenta are

$$
\left\langle A^{\mathrm{can}}\right\rangle=\left\langle\frac{\left|p_{z}\right| E_{z}-p_{z}^{2}}{2 E}\right\rangle_{+},\left\langle B^{\mathrm{can}}\right\rangle=\left\langle\frac{p_{\perp}^{2}\left|p_{z}\right|+p_{z}^{2} E_{z}}{4 E^{3} e_{z}}\right\rangle_{+}
$$

For $p \gg m$ they differ by a factor $\frac{1}{2} \frac{|p|}{\left|p_{z}\right|}$ from the ones for kinetic momenta (6.82). 
The generated baryon asymmetry depends on the squark spectrum because of the potential suppression due to strong sphalerons. In the following we consider four different squark spectra which are listed in table 2. Case A corresponds to the massless limit, where strong sphaleron suppression is most efficient. In case B all squarks are assumed to be heavy, with exception of the right-handed stop, which is taken massless. The spectra $\mathrm{C}$ and D are obtained from the universal pattern of SUSY breaking discussed in section 2. The first and second generation squarks are almost degenerate in mass with $\tilde{b}_{2}$, thus $2 k_{B}=k_{Q_{1}}=k_{Q_{2}}=2 k_{U}=\ldots$ Case $\mathrm{C}$ corresponds to the example of explicit CP-violation discussed in context of fig. 6, while case D is the squark spectrum of the transitionally CP-violating example of fig. 7 .

According to eq. (6.75) the produced baryon asymmetry is proportional to the parameter $C$, which is related to the chemical potential of left-handed quarks (6.66) and also given in table 2. It turns out that the results for different spectra can indeed be obtained by rescaling with the relevant $C$ parameters, i.e. the indirect impact of the statistical factors in the diffusion equations (6.64) is small. For the massless case A, and more general for the case of degenerate squarks, $C$ vanishes. Baryon production is completely suppressed by rapid strong sphalerons transitions, which is a well known result [75]. The cancellation disappears for non-degenerate squarks. Even for the realistic spectra $\mathrm{C}$ and D, resulting from universal SUSY breaking, there is only a mild suppression by a factor of 3-4 relative to the idealized spectrum B (usually assumed in context of the MSSM).

To begin with let us consider the source term of the top quarks (6.79), which in the first place depends on the variation of the phase of the top quark mass along the wall, $S_{t} \propto \delta \theta_{t}$, and the wall thickness $L_{w}$. From eqs. (5.14) and (5.22) we obtain $\delta \theta_{t}=\delta \theta_{2}=\cos ^{2}(\beta) \delta \theta$, where $\theta_{2}$ denotes the phase of $H_{2}^{0}$ and $\theta$ is the (gauge invariant) sum of the phases of the two Higgs fields, that entered the bubble equations in section 5. As a consequence, $S_{t}$ is rather sensitive to the Higgs vev ratio. Baryon production from the top quark source is particularly efficient for small values of $\tan \left(\beta_{T}\right)$. In fig. 9 we present the baryon asymmetry induced by $S_{t}$ as a function of the wall thickness and velocity, where we used $v_{c} / T_{c}=1.60, T_{c}=101 \mathrm{GeV}$ and $y_{t}=1.015$ (corresponding to $\tan \beta=5$ ).

Our resultst四 would be slightly enhanced by larger values of $v_{c} / T_{c}$. We measure the

\footnotetext{
${ }^{14}$ In numerical evaluations we still used the brackets $\left\langle A^{\text {can }}\right\rangle,\left\langle B^{\text {can }}\right\rangle$ of eq. (6.83) instead of $\langle A\rangle,\langle B\rangle$ of eqs. (6.82) in order not to be forced to completely repeat the whole analysis. This introduces a deviation of order (1), in the case $|p| \gg m$ a factor $\frac{1}{2}$ instead of $\frac{\left|p_{z}\right|}{|p|}$ in the brackets $\langle A\rangle$ and $\langle B\rangle$. This is well within the accuracy of the present investigation.
}

\begin{tabular}{|c|c|c||c|c|c|c||c|c|c|c|c|}
\hline & $M_{0}$ & $A_{0}$ & $M_{\tilde{t}_{1}}$ & $M_{\tilde{t}_{2}}$ & $M_{\tilde{b}_{2}}$ & $M_{\tilde{u}}$ & $k_{Q_{3}}$ & $k_{T}$ & $k_{B}$ & $k_{Q_{1}}$ & $C$ \\
\hline $\mathrm{A}$ & & & 0 & 0 & 0 & 0 & 18 & 9 & 9 & 18 & 0 \\
\hline $\mathrm{B}$ & & & $\infty$ & 0 & $\infty$ & $\infty$ & 6 & 9 & 3 & 6 & 0.385 \\
\hline $\mathrm{C}$ & 125 & -100 & 418 & 238 & 334 & 375 & 7.26 & 4.56 & 3.60 & 7.20 & 0.125 \\
\hline $\mathrm{D}$ & 100 & -100 & 371 & 201 & 217 & 325 & 8.43 & 4.98 & 3.87 & 7.74 & 0.102 \\
\hline
\end{tabular}

Table 2: Squark spectra and corresponding statistical factors used in the discussion of the baryon asymmetry. For the sets $\mathrm{C}$ and $\mathrm{D}$ we used $\lambda=0.05, k=0.4, m_{0}=200 \mathrm{GeV}$ and $\tan \beta=-5$. Furthermore, we took $x=-100(-150) \mathrm{GeV}$ in case $\mathrm{C}(\mathrm{D})$. The sets $\mathrm{A}$ and $\mathrm{B}$ do not follow the universal pattern of SUSY breaking considered in section 2. (Units in $\mathrm{GeV}$.) 


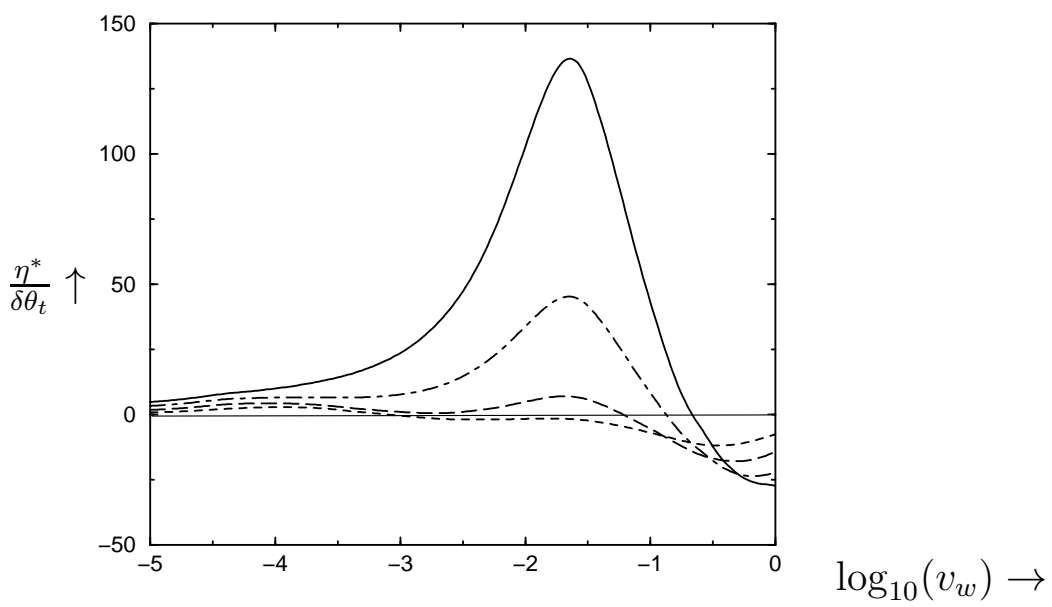

Figure 9: Top quark contribution to the baryon asymmetry for the spectrum $\mathrm{D}$ in units of $2 \times 10^{-11}$ normalized by $\delta \theta_{t}$ as a function of the wall velocity for different values of the wall thickness $L_{w}=20 / T, 10 / T, 5 / T, 3 / T$ (from below).

baryon asymmetry in units of $2 \times 10^{-11}$, which is the lower observational bound 69

$$
\eta^{*}=\frac{\eta}{2 \times 10^{-11}} .
$$

In fig. 9 we display the dependence of the baryon asymmetry on the wall velocity for different values of the wall thickness $L_{w}=20 / T, 10 / T, 5 / T, 3 / T$. We used the squark spectrum D in the evaluation. Results for other spectra can be easily obtained by rescaling with the relevant $C$ parameters of table 2 2 . For $L_{w}<\sim 10 / T$ baryon production is most efficient for $v_{w} \sim 0.02-0.03$. Interesting enough, this is precisely the range of wall velocities found in recent calculations in the MSSM [66, 68]. $\eta$ becomes small for large values of $v_{w}$ because transport in front of the wall gets more and more inefficient. For small wall velocities deviation from equilibrium becomes too small to generate a relevant baryon asymmetry. We observe an approximate $1 / L_{w}^{2}$ dependence in the generated baryon number. This behavior is expected from the explicit expression for the source term (6.79), which contains three derivatives with respect to $\bar{z}$. Since the calculation of $\eta$ (6.75) requires one (numerical) integration of the source over $\bar{z}^{\prime}$, approximating $\partial_{\bar{z}} \sim 1 / L_{w}$ we obtain $\eta \propto 1 / L_{w}^{2}$. As a result baryon production from the top quark source is most efficient in the case of slow $\left(v_{w} \sim 10^{-2}\right)$ and thin walls, and for non-degenerate squark spectra. It turns out that the Higgs source term behaves in a similar way. In fig. 9 we assumed $\delta \beta=10^{-3}$ in the bubble wall which is a typical value according to section 4 . However, even large values, $\delta \beta=10^{-2}$, change the result only by a few percent. From fig. 9 we approximately obtain for the maximal value of the baryon asymmetry

$$
\eta_{\max }^{*} \sim 47\left(\frac{5}{L_{w} T}\right)^{2} \delta \theta_{t} .
$$

However, for medium and large Higgs vev ratios even small values of $\delta \theta_{t} \sim \mathcal{O}\left(10^{-2}\right)$ are difficult to achieve, since $\delta \theta_{t}=\cos ^{2}(\beta) \delta \theta$. In the case of $\tan \beta=5$, for instance, we obtain $\delta \theta_{t}=\delta \theta / 26$, which leads to a significant suppression. In the example of transitional CP-violation (fig. (7) we have $\tan \beta=5, L_{w}=3$ and $\delta \theta \sim 1 / 20$ leading to $\eta_{\max }^{*} \sim 1 / 4$. 

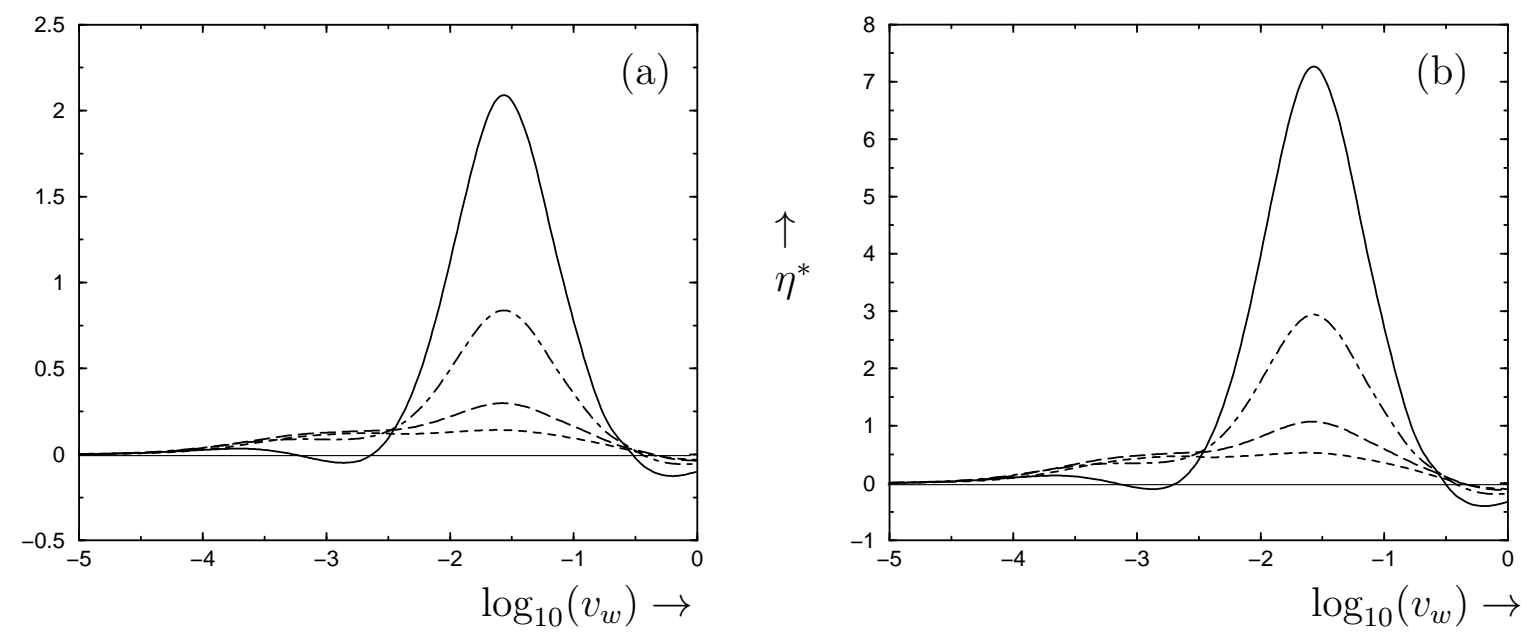

Figure 10: The chargino contribution to the baryon asymmetry in units of $2 \times 10^{-11}$ as a function of the wall velocity for different values of the wall thickness $L_{w}=$ $20 / T, 10 / T, 5 / T, 3 / T$ (from below). We use the squark spectrum $\mathrm{C}$ and the example of explicit CP-violation considered in the context of fig. 6. (b): The same quantity for the transitionally CP-violating bubble wall of fig. 7 and the squark spectrum D.

In case of explicit CP-violation, the top quark contribution to the baryon asymmetry is much smaller even, and can be safely neglected.

We now come to the charged Higgsino contribution to the baryon asymmetry fueled by the source terms (6.80) and (6.81) for the examples of explicit and transitional CP-violation already discussed in the context of figs. 6 and 7. Again, we present our results $^{14}$ as a function of the wall velocity for different values of the wall thickness $L_{w}=20 / T, 10 / T, 5 / T, 3 / T$. Concerning the dependence of the generated baryon asymmetry on the wall velocity and the wall thickness we find a similar behavior as in the case of the top quark source: $\eta$ becomes large for thin walls and $v_{w} \sim 10^{-2}$.

The baryon asymmetry generated from the chargino dispersion relation is shown in fig. 10. If the top Yukawa interactions were in equilibrium, as was assumed in elder work on the subject, this contribution is completely erased, because of the equal source terms for both Higgsinos (6.80,6.81). Even with finite top Yukawa rates no baryon asymmetry is generated from this source, if both Higgs fields have equal rates in the diffusion equations (6.64), i.e. if $\Gamma_{y}+\Gamma_{y A}=\Gamma_{y \mu}$ and $\Gamma_{H_{1}}=\Gamma_{H_{2}}$. In our scenario an asymmetry in the top Yukawa rates is inevitably induced by the heavy left-handed stop, leading to a strong suppression of $\Gamma_{y \mu}$ (6.77). In the evaluations of fig. 10 we used $\delta \beta=10^{-3}$. If we increase the change in the Higgs vev ratio by a factor of 10 , the results only change by about 15 percent.

From fig. 10a for the case of explicit CP-violation we read of

$$
\eta_{\max }^{*} \sim 0.8\left(\frac{5}{L_{w} T}\right)^{2} \sin \left(\phi_{\mu}\right) .
$$

Thus CP-violating phases of order $10^{-1}$ are required to account for the observed baryon asymmetry in this scenario. Phases of this size are compatible with the EDM experiments only if the first and second generation squarks are heavy or in the case of accidental cancellations. Our findings for baryogenesis from explicit CP-violation in the NMSSM 

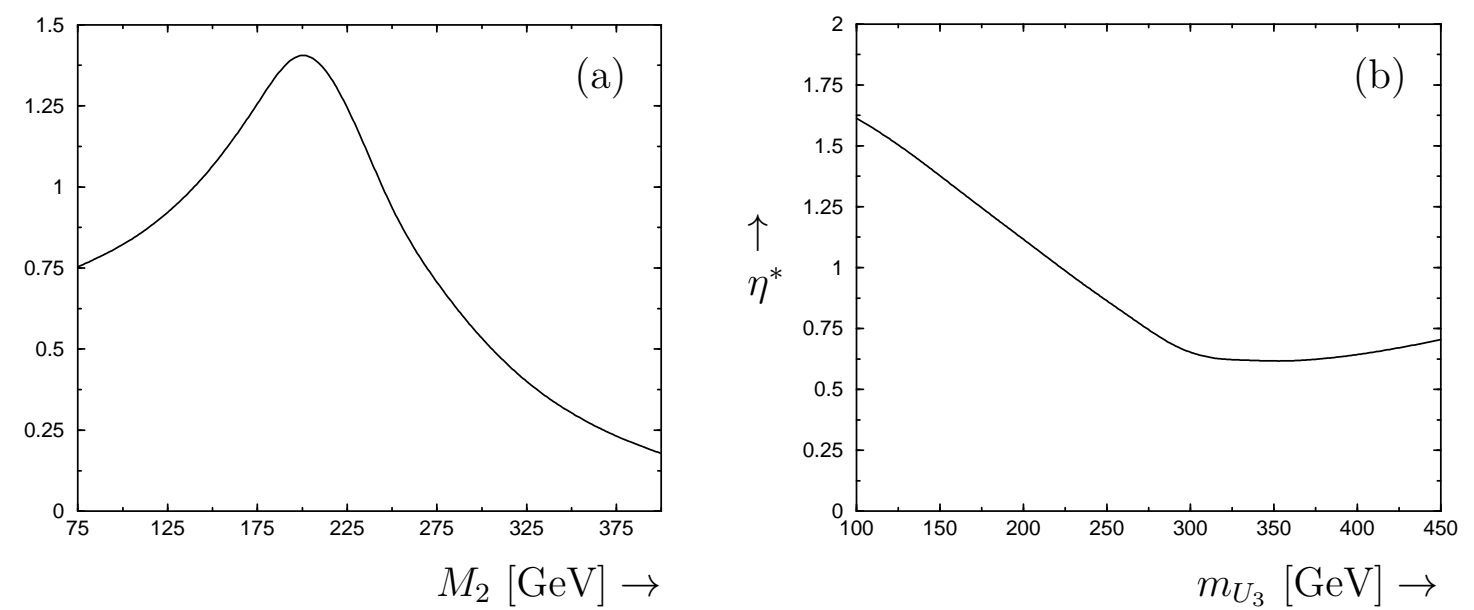

Figure 11: Chargino contribution to the baryon asymmetry in units of $2 \times 10^{-11}$ as a function of the gaugino mass parameter $M_{2}$ (a) and as a function of the soft scalar mass parameter $m_{U_{3}}$. In both cases we otherwise used the parameter set of explicit CP-violation (fig. 6) $, \phi_{\mu}=0.1, L_{w}=5 / T, v_{w}=0.03$ and $\delta \beta=10^{-3}$.

are very similar to the results in the MSSM [73]. Still, baryogenesis in the NMSSM is slightly more effective because of the thinner bubble walls.

The scenario of transitional CP-violation is a very attractive alternative, being not in conflict with the EDM experiments. As shown in fig. 10a, it can generate the baryon asymmetry for a considerable range of wall velocities, especially for $L_{w}=3 / T$. It even allows for a certain baryon "over-production" to compensate for a partial washout of the generated baryon asymmetry due to the existence of bubbles with opposite CP-properties. The introduction of small explicit phases lifts the energetic degeneracy of the two sort of bubbles, leading to a more rapid nucleation of bubbles of the true vacuum and a net baryon production.

Up to now we centered the discussion of baryon production around two particular sets of NMSSM parameters. However, the results may considerably change if different regions in the parameter space are considered. Of course, baryon production becomes inefficient if the particles which supply the CP-violating source terms, in the first place the Higgsinos, are heavy and decouple from the plasma. The thermal averages, which enter the sources (6.79) - 6.81), already decrease by a factor of 10 if $m / T$ is raised from one to five. In case of $m / T=10$ the suppression is of order $\mathcal{O}\left(10^{-3}\right)$ and successful baryogenesis becomes extremely difficult. If universal SUSY breaking is assumed this occurs in the regime $M_{0} \gtrsim 700 \mathrm{GeV}$.

The chargino source term is rather sensitive to the relative size of the Higgsino mass parameter, $\mu$, and the mass of the $\mathrm{SU}(2)$-gauginos, $M_{2}$. In fig. 117a we present the dependence of the generated baryon asymmetry on $M_{2}$, while the remaining parameters as well as the bubble wall profile are taken from the example of explicit CP-violation considered in fig. 6, i.e. the effect of a change in $M_{2}$ on the bubble wall is ignored. Moreover, we use the squark spectrum $\mathrm{C}, \phi_{\mu}=0.1 L_{w}=5 / T$ and $v_{w}=0.03$. We find a resonance structure for $\eta$, with the peak located at $M_{2} \sim|\mu|=226 \mathrm{GeV}$. Fig. 10a corresponds to $M_{2}=103$ $\mathrm{GeV}$. In fig. 11 b we present the dependence of the baryon asymmetry on the soft breaking scalar mass parameter $m_{U_{3}}$. Even though this parameter has no direct impact on the chargino source terms (6.80), (6.81) it alters the statistical factors $k_{Q_{3}}$ and $k_{T}$ due to 
varying stop masses. Baryogenesis becomes less efficient if the two stop stated are more or less degenerate. In the case under consideration this happens for $m_{U_{3}} \sim m_{Q_{3}}=329$ GeV. In fig. 10a we used $m_{U_{3}}=256 \mathrm{GeV}$.

\section{Conclusions}

In our computation we used the semi-classical approximation to describe the interaction of the particles in the plasma and the propagating bubble wall which in context of the MSSM has been introduced in ref. [64]. Our work differs in various aspects from that analysis. We included contributions from top quarks which have previously been neglected and took into account also position-dependent mixing in the chargino mass matrix ("flavor contribution"). In ref. [64 different results were obtained due to an error in the transport equations, which prevented the cancellation between the dominating helicity parts in the $\tilde{h}_{1}^{-}$and $\tilde{h}_{2}^{+}$source terms. With dispersion relations for canonical momenta, the charged Higgsinos provide in the MSSM CP-violating source terms which are proportional to the variation of $\tan \beta$ in the bubble wall. This result would agree well with refs. [49, 52, 58] where different methods where used to determine the CP-violating source terms. Recently $\delta \beta$ independent contributions to the source terms in the MSSM have been calculated 660. However, these arise from higher orders in a Higgs insertion expansion and should not be included in our semi-classical approach. In the NMSSM non-vanishing source terms for stops and charginos (and for the top quark) are generated even for constant $\tan \beta$ because of the changing singlet field, and due to CP-violating bubble walls. These arise either from explicit CP-violation, which in case of the NMSSM is possible already in the tree-level Higgs potential, or from transitional CP-violation.

Using kinetic momenta in the dispersion relations as suggested convincingly in ref. [64] $\tilde{h}_{1}$ and $\tilde{h}_{2}$ sources are exactly equal and $\tan \beta^{\prime}$ effects cancel. Thus one has to consider a source symmetric in the Higgses. This contribution can be also sizeable, if the Yukawa interactions are not in equilibrium [64] and if the left-handed stop is heavy [73, 74]. This statement ist unchanged in our singlet model. The WKB results and their difference to other work should be further discussed using quantum transport equations.

In our work we investigated the impact of different squark spectra on the emerging baryon asymmetry, while in ref. [64] only the massless approximation was considered. As a consequence, we obtained a non-vanishing baryon asymmetry, while assuming the strong sphalerons to be in equilibrium. Baryogenesis becomes most efficient for non-degenerate squarks. The generated baryon asymmetry is roughly proportional to $1 / L_{w}^{2}$. Since in the NMSSM the bubble walls can be considerably thinner than in the MSSM, more baryons can be produced. The baryon asymmetry strongly depends on the velocity of the bubble wall, which in the NMSSM has not been computed so far. Interesting enough, we find that the maximal baryon number is generated for $v_{w} \sim 10^{-2}$. This is a typical value for the wall velocity in the MSSM.

Finally, we studied the dependence of the chargino source terms on $\mu, M_{2}, m_{Q_{3}}^{2}$ and $m_{U_{3}}^{2}$. We found an enhancement if $\mu$ and $M_{2}$ become degenerate. This resonance behavior has also been reported in refs. [49, 52, 60, 64. If $m_{Q_{3}}^{2} \sim m_{U_{3}}^{2}$, baryon production is partially suppressed by strong sphalerons. Concluding, the NMSSM can account for the observed baryon asymmetry, especially if $\mu \sim M_{2}$ and if the squark spectrum is rather non-degenerate, or if transitional CP-violation occurs. Both scenarios point to a 
non-universal pattern of SUSY breaking.

\section{Acknowledgment}

We thank P. John for useful discussions. This work was supported in part by the TMR network Finite Temperature Phase Transitions in Particle Physics, EU contract no. ERBFMRXCT97-0122.

\section{References}

[1] K. Kajantie, M. Laine, K. Rummukainen, M. E. Shaposhnikov, Phys. Rev. Lett. 77 (1996) 2887.

[2] D. Bödeker, P. John, M. Laine, M. G. Schmidt, Nucl. Phys. B497 (1997) 387; M. Carena, M. Quiros, C. E. M. Wagner, Nucl. Phys. B523 (1998) 3; M. Losada, Nucl. Phys. B537 (1999) 3.

[3] M. Laine, K. Rummukainen, Nucl. Phys. B535 (1998) 423.

[4] See e.g., A. Pilaftsis, C. E. M. Wagner, Nucl. Phys. B553 (1999) 3.

[5] S. J. Huber, P. John, M. Laine, M. G. Schmidt, Phys. Lett. B475 (2000) 104.

[6] H.-P. Nilles, M. Srednicki, D. Wyler, Phys. Lett. B120 (1983) 346; J.-M. Frere, D. R. T. Jones, S. Raby, Nucl. Phys. B222 (1983) 11; L. E. Ibanez, J. Mas, Nucl. Phys. B286 (1987) 107.

[7] S. A. Abel, S. Sarkar, P. L. White, Nucl. Phys. B454 (1995) 663.

[8] M. Pietroni, Nucl. Phys. B402 (1993) 27.

[9] A. T. Davies, C. D. Froggatt, R. G. Moorhouse, Phys. Lett. B372 (1996) 88.

[10] See e.g., N. Polonsky, proceedings of Supersymmetry, supergravity and superstring, Seoul 1999, 100-124 hep-ph/9911329.

[11] U. Ellwanger, Phys. Lett. B133 (1983) 187; J. Bagger, E. Poppitz, L. Randall, Nucl. Phys. B455 (1995) 59.

[12] S. A. Abel, Nucl. Phys. B480 (1996) 55.

[13] C. Panagiotakopoulos, K. Tamvakis, Phys. Lett. B446 (1999) 224.

[14] S. A. Abel, hep-ph/9603301.

[15] U. Ellwanger, Phys. Lett. B349 (1995) 57; H.-P. Nilles, N. Polonsky, Phys. Lett. B412 (1997) 69; A. de Gouvea, A. Friedland, H. Murajama, Phys. Rev. D57 (1998) 5676; T. Han, D. Marfatia, R. -J. Zhang, Phys. Rev. D61 (2000) 013007.

[16] S. J. Huber, M. G. Schmidt, Eur. Phys. J. C10 (1999) 473; S. J. Huber, proceedings of Strong and electroweak matter, Copenhagen 1998, 339-343 hep-ph/9902325. 
[17] J.-P. Derendinger, C. A. Savoy, Nucl. Phys. B237 (1984) 307.

[18] S. Martin, M. Vaughn, Phys. Rev. D50 (1994) 2282.

[19] U. Ellwanger, M. Rausch de Traubenberg, C. A. Savoy, Nucl. Phys. B492 (1997) 21.

[20] L. Alvarez-Gaume, J. Polchinski, M. Wise, Nucl. Phys. B221 (1983) 495.

[21] U. Ellwanger, C. Hugonie, Phys. Lett. B457 (1999) 299.

[22] S. Abel, C. A. Savoy, Nucl. Phys. B532 (1998) 3.

[23] For a summary see, e.g., C. Caso et al., Particle Data Group, Eur. Phys. J. C3 (1998) 1.

[24] ALEPH Collaboration (R. Barate et al.), CERN-EP/99-014.

[25] J. Ellis, J. Gunion, H. Haber, L. Roszkoswki, F. Zwirner, Phys. Rev. D39 (1989) 844; U. Ellwanger, M. Rausch de Traubenberg, C. A. Savoy, Phys. Lett. B315 (1993) 331; U. Ellwanger, C. Hugonie, hep-ph/9909260.

[26] OPAL Collaboration (G. Abbiendi et al.), Eur. Phys. J. C7 (1999) 407.

[27] P. Brax, U. Ellwanger, C. A. Savoy, Phys. Lett. B347 (1995) 269.

[28] L. Dolan, R. Jackiw, Phys. Rev. D9 (1974) 3320.

[29] M. Dine, R. G. Leigh, P. Huet, A. Linde, D. Linde, Phys. Rev. D46 (1992) 550.

[30] M. E. Carrington, Phys. Rev. D45 (1992) 2933; J. R. Espinosa, M. Quiros, F. Zwirner, Phys. Lett. B314 (1993) 206.

[31] A. D. Linde, Phys. Lett. B70 (1977) 306; Nucl. Phys. B216 (1983) 421.

[32] L. McLerran, M. E. Shaposnikov, N. Turok, M. Voloshin, Phys. Lett. B256 (1991) 451.

[33] P. John, Phys. Lett. B452 (1999) 221.

[34] P. John, proceedings of Strong and electroweak matter, Copenhagen 1998, 349-353 hep-ph/9901326.

[35] J.M. Moreno, M. Quirós, M. Seco, Nucl. Phys. B526 (1998) 489.

[36] J. M. Cline, G. D. Moore, G. Servant, Phys. Rev. D60 (1999) 105035.

[37] P. John, PhD-Thesis, University of Heidelberg, 1999.

[38] W. Buchmüller, D. Wyler, Phys. Lett. B121 (1983) 321; J. Polchinski, M. B. Wise, Phys. Lett. B125 (1983) 393.

[39] I. S. Altraev et al., Phys. Lett. B276 (1992) 242; E. Commins et al., Phys. Rev. A50 (1994) 2960. 
[40] Y. Kizukuri, N. Oshimo, Phys. Rev. D45 (1992) 1806.

[41] T. Ibrahim, P. Nath, Phys. Rev. D58 (1998) 111301; M. Brhlik, G. J. Good, G. L. Kane, Phys. Rev. D59 (1999) 115004.

[42] A. Pomerol, Phys. Lett. B287 (1992) 331.

[43] M. Matsuda, M. Tanimoto, Phys. Rev. D52 (1995) 3100.

[44] K. S. Babu, S. M. Barr, Phys. Rev. D49 (1994) 2156; N. Haba, M. Matsuda, M. Tanimoto, Phys. Rev. D54 (1996) 6928.

[45] A. T. Davies, C. D. Frogatt, A. Usai, Proc. of the International Europhysics Conference on High Energy Physics, Jerusalem (1997), Eds. D. Lellouch, G. Mikenberg, E. Rabinovic, Springer-Verlag (1998), p891, [hep-ph/9712501]; proceedings of Strong and electroweak matter, Copenhagen 1998, 227-231 [hep-ph/9902476.

[46] D. Comelli, M. Pietroni, A. Riotto, Phys. Rev. D50 (1994) 7703.

[47] D. Comelli, M. Pietroni, Phys. Lett. B306 (1993) 67; J. R. Espinosa, J. M. Moreno, M. Quiros, Phys. Lett. B319 (1993) 505; K. Funakubo, A. Kakuto, S. Otsuki, F. Toyoda, Prog. Theor. Phys. 99 (1998) 1045.

[48] K. Funakubo, A. Kakuto, S. Otsuki, F. Toyoda, Prog. Theor. Phys. 102 (1999) 389.

[49] M. Carena, M. Quirós, A. Riotto, I. Vilja, C. E. M. Wagner, Nucl. Phys. B503 (1997) 387.

[50] For reviews see e.g. P. A. Henning, Phys. Rep. 253 (1995) 235; C. Greiner, S. Leupold, Annals Phys. 270 (1998) 328.

[51] L. P. Kadanoff, G. Baym, Quantum Statistical Mechanics, Benjamin Ed., New York, 1962.

[52] A. Riotto, Phys. Rev. D53 (1996) 5834; Phys. Rev. D58 (1998) 095009.

[53] A. G. Cohen, D. B. Kaplan, A. E. Nelson, Phys. Lett. B245 (1990) 561; Nucl. Phys. B349 (1991) 727; A. E. Nelson, D. B. Kaplan, A. G. Cohen, Nucl. Phys. B373 (1992) 453.

[54] K. Funakubo, A. Kakuto, S. Otsuki, K. Takenaga, F. Toyoda, Phys. Rev. D50 (1994) 1105.

[55] M. Joyce, T. Prokopec, N. Turok, Phys. Rev. Lett. 75 (1995) 1695; Phys. Rev. D53 (1996) 2930.

[56] M. Aoki, N. Oshimo, A. Sugamoto, Progr. Theor. Phys. 98 (1997) 1179; ibid. 98 (1997) 1325.

[57] H. Davoudiasl, K. Rajagopal, E. Westphal, Nucl. Phys. B515 (1998) 384.

[58] P. Huet, A. E. Nelson, Phys. Rev. D53 (1996) 4597. 
[59] M. B. Gavela, P. Hernandez, J. Orloff, O. Pene, C. Quimbay, Nucl. Phys. B430 (1994) 382; P. Huet, E. Sather, Phys. Rev. D51 (1995) 379.

[60] N. Rius, V. Sanz, Nucl. Phys. B570 (2000) 155.

[61] N. Turok, J. Zadrozny, Nucl. Phys. B358 (1991) 471.

[62] A. G. Cohen, D. B. Kaplan, A. E. Nelson, Phys. Lett. B263 (1991) 86.

[63] M. Joyce, T. Prokopec, N. Turok, Phys. Rev. D53 (1996) 2958.

[64] J. M. Cline, M. Joyce, K. Kainulainen, Phys. Lett. B417 (1998) 79.

[65] M. Joyce, K. Kainulainen, T. Prokopec, Phys. Lett. B468 (1999) 128; proceedings of Strong and electroweak matter, Copenhagen 1998, 232-237 [hep-ph/9906413]; JHEP 0010 (2000) 029.

[66] G. D. Moore, JHEP 0003 (2000) 006.

[67] G. Moore, T. Prokopec, Phys. Rev. D52 (1995) 7182.

[68] P. John, M. G. Schmidt, hep-ph/0002050.

[69] K. Olive, astro-ph/9901231.

[70] A. G. Cohen, D. B. Kaplan, Phys. Lett. B199 (1987) 257; Nucl. Phys. B308 (1988) 913; A. G. Cohen, D. B. Kaplan, A. E. Nelson, Phys. Lett. B336 (1994) 41.

[71] J. M. Cline, M. Joyce, K. Kainulainen, JHEP 0007 (2000) 018; K. Kainulainen, hep-ph/0002273; M. J. Cline, K. Kainulainen, Phys. Rev. Lett. 85 (2000) 5519.

[72] J. M. Cline, K. Kainulainen, Phys. Lett. B356 (1995) 19.

[73] S. J. Huber, P. John, M. G. Schmidt, hep-ph/0101249.

[74] M. Carena, J. M. Moreno, M. Quiros, M. Seco, C. E. M. Wagner, hep-ph/0011055.

[75] G. F. Giudice, M. Shaposhnikov, Phys. Lett. B326 (1994) 118.

[76] G. D. Moore, Phys. Lett. B412 (1997) 359; G. D. Moore, N. Turok, Phys. Rev. D56 (1997) 6533.

[77] D. Bödeker, Phys. Lett. B426 (1998) 351. 